Interfaces and Free Boundaries 16 (2014), 41-64

DOI 10.4171/IFB/313

\title{
Stability and bifurcation of equilibria for the axisymmetric averaged mean curvature flow
}

\author{
JEREMY LECRONE \\ Department of Mathematics, Kansas State University, Manhattan, KS, USA \\ E-mail: lecronjs@ksu.edu
}

[Received 8 November 2012 and in revised form 7 December 2013]

\begin{abstract}
We study the averaged mean curvature flow, also called the volume preserving mean curvature flow, in the particular setting of axisymmetric surfaces embedded in $\mathbb{R}^{3}$ satisfying periodic boundary conditions. We establish analytic well-posedness of the flow within the space of little-Hölder continuous surfaces, given rough initial data. We also establish dynamic properties of equilibria, including stability, instability, and bifurcation behavior of cylinders, where the radius acts as a bifurcation parameter.
\end{abstract}

2010 Mathematics Subject Classification: Primary 35K93, 53C44; Secondary 35B35, 35B32.

Keywords: Averaged mean curvature flow, periodic boundary conditions, maximal regularity, nonlinear stability, bifurcation.

\section{Introduction}

The averaged mean curvature flow is a second-order geometric evolution law, acting on closed, compact, connected, sufficiently smooth hypersurfaces $\Gamma$ immersed in $\mathbb{R}^{n}$. The evolution of $\Gamma$ involves the (normalized) mean curvature $\mathcal{H}(\Gamma)$, which is simply the sum of the principal curvatures on the surface. In particular, one seeks to find a one-parameter family of smooth, immersed, orientable hypersurfaces $\{\Gamma(t): t \geqslant 0\}$ which satisfy the evolution equation

$$
\left\{\begin{array}{l}
V(t)=h(\Gamma(t))-\mathcal{H}(\Gamma(t)), \quad t>0, \\
\Gamma(0)=\Gamma_{0},
\end{array}\right.
$$

where $V(t)$ denotes the normal velocity of the hypersurface $\Gamma(t), \Gamma_{0}$ is a given initial surface, and $h(\Gamma)$ is the integral average of the mean curvature $\mathcal{H}(\Gamma)$ on $\Gamma$. Two important features of the flow (1.1) are that the surface area of $\Gamma(t)$ is decreasing in $t$ and the (signed) volume enclosed by the surface $\Gamma(t)$ is preserved, as long as smooth solutions exist. These features provide a starting point for our analysis of the dynamical properties of solutions.

Problem (1.1) is a modification of the mean curvature flow, which is the related evolution equation without the integral average term $h(\Gamma)$ in the governing equation. These two problems have a long and rich history of investigation which we do not attempt to summarize here. We will simply highlight some of the important historical results which are relevant to our current investigation.

Both the mean curvature and averaged mean curvature flows have been considered in a wide range of settings for $\Gamma$. Cases considered include curves in $\mathbb{R}^{2}$, [15, 16], hypersurfaces immersed in $\mathbb{R}^{n},[5,14,18,26]$, and immersed submanifolds of smooth Riemannian manifolds $\left(m^{n}, g\right)$, [19], for arbitrary dimensions $n \geqslant 2$. 
The first well-posedness results for (1.1) were established by Gage [15], in the setting of plane curves $\gamma \subset \mathbb{R}^{2}$. Under convexity assumptions on the initial curve $\gamma_{0}$, it is shown that solutions are global, convex for all time $t>0$, and solutions converge to a circle. These results were later generalized by Huisken [18], who proved existence of global solutions assuming smooth uniformly convex initial hypersurfaces in $\mathbb{R}^{n}$. It is also shown in this setting that solutions converge to an $n$-dimensional sphere. Escher and Simonett [14] later proved well-posedness for rough initial data (in the little Hölder spaces $h^{1+\beta}$ ), including the possibility of nonconvex hypersurfaces, and further proved that the family of $n$-dimensional spheres is locally exponentially attractive in the topology of $h^{1+\beta}$. Regarding further qualitative properties of solutions, Mayer and Simonett [26] proved the first analytic results regarding hypersurfaces evolving according to (1.1) which lose embeddedness.

Considering axisymmetric, or rotationally symmetric, surfaces, several authors have investigated both the mean curvature and averaged mean curvature flows in this setting, cf. [5, 6] and references therein. Of particular note is a result of Athanassenas [5], in the setting of smooth surfaces satisfying Neumann boundary conditions, regarding global existence and convergence to a cylinder for given initial surfaces satisfying an isoperimetric-type inequality. A recent article of Hartley [17] refines this result to rough initial data, also allowing for non-rotationally symmetric initial surfaces. An exponential convergence rate is also established, given initial data $h^{1+\beta}$-close to a cylinder, using techniques similar to [14].

In the current article, we develop a function space setting adequate to establish well-posedness of the axisymmetric averaged mean curvature flow with rough initial data and to investigate geometric properties of solutions analytically. The methods of the paper also allow for application to higherorder nonlinear problems. For example the author and Simonett [23] recently applied similar methods to derive results for the surface diffusion flow, a fourth-order geometric evolution law, also in the setting of axisymmetric surfaces with periodic boundary conditions.

We conclude the introduction with a brief outline of the article. In Section 2, we define appropriate spaces and introduce analytic tools that will be used throughout the paper. In Section 3, we take advantage of maximal regularity properties for the governing operator of (2.4), in conjunction with a quasilinear structure, in order to establish well-posedness and regularity of solutions. We are then able to explicitly characterize all equilibria (stationary solutions) to the problem in Section 4, using results regarding closed surfaces of revolution with constant mean curvature.

After characterizing equilibria, the remainder of the paper is dedicated to investigating properties of two particular families of equilibria, namely the cylinders of radius $r_{\star}>0$ and families of $\frac{2 \pi}{k}$ periodic unduloids. In Section 5, we develop nonlinear stability and instability results for cylinders, where the size of the radius governs the stability of the cylinder. One will see in this section how maximal regularity provides a crucial connection to tools in nonlinear functional analysis, including applications of the Implicit Function Theorem on Banach spaces. We conclude the article with an investigation of bifurcations of equilibria, in particular we use analytic methods to establish the qualitative structure of the intersections between the family of unduloids and the family of cylinders.

\section{Notation and conventions}

For the remainder of the paper, we consider the case of $\Gamma \subset \mathbb{R}^{3}$ an embedded surface which is symmetric about an axis of rotation (which we take to be the $x$-axis, without loss of generality) and satisfies prescribed periodic boundary conditions on some fixed interval $L$ of periodicity (we take $L=[-\pi, \pi]$ and enforce $2 \pi$ periodicity for convenience; see Remark 6.2 below for 
comments regarding extension of our setting to arbitrary intervals of periodicity). In particular, the axisymmetric surface $\Gamma$ is characterized by the parametrization

$$
\Gamma=\{(x, r(x) \cos (\theta), r(x) \sin (\theta)): x \in \mathbb{R}, \theta \in[-\pi, \pi]\},
$$

where the function $r: \mathbb{R} \rightarrow(0, \infty)$ is the profile function for the surface $\Gamma$. Conversely, a profile function $r: \mathbb{R} \rightarrow(0, \infty)$ generates an axisymmetric surface $\Gamma=\Gamma(r)$ via the parametrization given above. Utilizing this explicit parametrization for axisymmetric surfaces, we can recast the averaged mean curvature flow as an evolution equation for the time-dependent profile functions $r=r(t, x)$.

Given a surface $\Gamma(r)$, it follows that the mean curvature is $\mathcal{H}(r)=\kappa_{1}+\kappa_{2}$, where

$$
\kappa_{1}=\frac{1}{r \sqrt{1+r_{x}^{2}}} \quad \text { and } \quad \kappa_{2}=\frac{-r_{x x}}{\left(1+r_{x}^{2}\right)^{3 / 2}}
$$

are the azimuthal and axial principle curvatures, respectively. Meanwhile, the normal velocity of $\Gamma=\Gamma(t)$ is

$$
V(t)=\frac{r_{t}}{\sqrt{1+r_{x}^{2}}},
$$

and, introducing the surface area functional

$$
S(r):=\int_{-\pi}^{\pi} r(x) \sqrt{1+r_{x}^{2}(x)} d x,
$$

which measures the surface area of one period of the induced axisymmetric surface $\Gamma(r)$ (modulo a factor of $2 \pi$ ), the integral average of the mean curvature is

$$
h(r)=\frac{1}{S(r)} \int_{-\pi}^{\pi} \mathcal{H}(r)(x) r(x) \sqrt{1+r_{x}^{2}(x)} d x .
$$

Defining the operator

$$
G(r):=\sqrt{1+r_{x}^{2}}[h(r)-\mathcal{H}(r)],
$$

we arrive at the expression

$$
\begin{cases}r_{t}(t, x)=G(r(t))(x), & x \in \mathbb{T}, t>0, \\ r(0)=r_{0}, & x \in \mathbb{T},\end{cases}
$$

for the periodic axisymmetric averaged mean curvature flow, where $\mathbb{T}:=[-\pi, \pi]$ is the onedimensional torus; with the points $-\pi$ and $\pi$ identified, endowed with the topology generated by the metric

$$
d_{\mathbb{T}}(x, y):=\min \{|x-y|, 2 \pi-|x-y|\}, \quad x, y \in \mathbb{T} .
$$

There is a natural relation between functions defined on $\mathbb{T}$ and $2 \pi$-periodic functions on $\mathbb{R}$, which the author explores in detail in the article [22]. Throughout the article, we will consider profile functions 
in the periodic little-Hölder spaces $h^{k+\alpha}(\mathbb{T})$, which are Banach spaces for $k \in \mathbb{N}_{0}:=\{0,1,2, \ldots\}$, $\alpha \in(0,1)$, defined by

$$
\begin{aligned}
h^{k+\alpha}(\mathbb{T}) & :={\overline{C^{\infty}(\mathbb{T})}}^{\|\cdot\|_{C^{k+\alpha}}(\mathbb{T})} \quad \text { and } \\
\|r\|_{h^{k+\alpha}} & :=\|r\|_{C^{k+\alpha}(\mathbb{T})}:=\sum_{j=0}^{k} \max _{x \in \mathbb{T}}\left|r^{(j)}(x)\right|+\sup _{\substack{x, y \in \mathbb{T} \\
x \neq y}} \frac{\left|r^{(k)}(x)-r^{(k)}(y)\right|}{d_{\mathbb{T}}^{\alpha}(x, y)},
\end{aligned}
$$

i.e., the closure of the smooth functions in the topology of the classic Hölder functions $C^{k+\alpha}$ over $\mathbb{T}$. If $\sigma \in \mathbb{R}_{+} \backslash \mathbb{Z}$ is fixed, then we denote by $h^{\sigma}(\mathbb{T})$ the little-Hölder space $h^{\lfloor\sigma\rfloor+\{\sigma\}}(\mathbb{T})$, where $\lfloor\sigma\rfloor$ denotes the largest integer not exceeding $\sigma$ and $\{\sigma\}:=\sigma-\lfloor\sigma\rfloor$.

In addition to functions defined on the one-dimensional torus, we consider standard classes of regular functions between Banach spaces. In particular, given Banach spaces $E, F$, and an open set $U \subset E$, we denote by $C^{k}(U, F)$ the class of $k$-times (Fréchet) differentiable functions mapping $U$ into $F$. We also denote by $C^{\omega}(U, F)$ the class of real-analytic functions, which are representable (in the topologies of $E$ and $F$ ) as a power series of $k$-linear maps from $E$ into $F$, cf. [10].

\subsection{Maximal regularity}

One essential tool that we use throughout the paper is the property of maximal regularity, also called optimal regularity in the literature. Maximal regularity has received considerable attention in connection with nonlinear parabolic partial differential equations, cf. [1-3, 7, 24, 28, 29]. Although maximal regularity can be developed in a more general setting, we will focus on the setting of continuous maximal regularity and direct the interested reader to the references [1,24] for a general development of the theory.

Let $\mu \in(0,1], J:=[0, T]$, for some $T>0$, and let $E$ be a (real or complex) Banach space. Following the notation of [7], we define spaces of continuous functions on $\dot{J}:=J \backslash\{0\}$ with prescribed singularity at 0 as

$$
\begin{aligned}
& B U C_{1-\mu}(J, E):=\left\{u \in C(\dot{J}, E):\left[t \mapsto t^{1-\mu} u(t)\right] \in B U C(\dot{J}, E)\right. \text { and } \\
& \left.\lim _{t \rightarrow 0^{+}} t^{1-\mu}\|u(t)\|_{E}=0\right\}, \quad \mu \in(0,1) \\
& \|u\|_{B_{1-\mu}}:=\sup _{t \in J} t^{1-\mu}\|u(t)\|_{E},
\end{aligned}
$$

where $B U C$ denotes the space consisting of bounded, uniformly continuous functions. We also define the subspace

$$
B U C_{1-\mu}^{1}(J, E):=\left\{u \in C^{1}(\dot{J}, E): u, \dot{u} \in B U C_{1-\mu}(J, E)\right\}, \quad \mu \in(0,1)
$$

and we set

$$
B U C_{0}(J, E):=B U C(J, E), \quad B U C_{0}^{1}(J, E):=B U C^{1}(J, E) .
$$

If $J=[0, a)$ for $a>0$, then we set

$$
\begin{array}{ll}
C_{1-\mu}(J, E):=\left\{u \in C(\dot{J}, E): u \in B U C_{1-\mu}([0, T], E),\right. & T<\sup J\}, \\
C_{1-\mu}^{1}(J, E):=\left\{u \in C^{1}(\dot{J}, E): u, \dot{u} \in C_{1-\mu}(J, E)\right\}, & \mu \in(0,1],
\end{array}
$$


which we equip with the natural Fréchet topologies induced by $B U C_{1-\mu}([0, T], E)$ and $B U C_{1-\mu}^{1}([0, T], E)$, respectively.

If $E_{1}$ and $E_{0}$ are a pair of Banach spaces such that $E_{1}$ is continuously embedded in $E_{0}$, denoted $E_{1} \hookrightarrow E_{0}$, we set

$$
\begin{aligned}
& \mathbb{E}_{0}(J):=B U C_{1-\mu}\left(J, E_{0}\right), \quad \mu \in(0,1], \\
& \mathbb{E}_{1}(J):=B U C_{1-\mu}^{1}\left(J, E_{0}\right) \cap B U C_{1-\mu}\left(J, E_{1}\right),
\end{aligned}
$$

where $\mathbb{E}_{1}(J)$ is a Banach space with the norm

$$
\|u\|_{\mathbb{E}_{1}(J)}:=\sup _{t \in \dot{J}} t^{1-\mu}\left(\|\dot{u}(t)\|_{E_{0}}+\|u(t)\|_{E_{1}}\right) .
$$

It follows that the trace operator $\gamma: \mathbb{E}_{1}(J) \rightarrow E_{0}$, defined by $\gamma v:=v(0)$, is well-defined and we denote by $\gamma \mathbb{E}_{1}$ the image of $\gamma$ in $E_{0}$, which is itself a Banach space when equipped with the norm

$$
\|x\|_{\gamma \mathbb{E}_{1}}:=\inf \left\{\|v\|_{\mathbb{E}_{1}(J)}: v \in \mathbb{E}_{1}(J) \text { and } \gamma v=x\right\} .
$$

Given $B \in \mathcal{L}\left(E_{1}, E_{0}\right)$, closed as an operator on $E_{0}$, we say $\left(\mathbb{E}_{0}(J), \mathbb{E}_{1}(J)\right)$ is a pair of maximal regularity for $B$ and write $B \in M R_{\mu}\left(E_{1}, E_{0}\right)$, if

$$
\left(\frac{d}{d t}+B, \gamma\right) \in \mathcal{L}_{i s o m}\left(\mathbb{E}_{1}(J), \mathbb{E}_{0}(J) \times \gamma \mathbb{E}_{1}\right), \quad \mu \in(0,1),
$$

where $\mathcal{L}_{\text {isom }}$ denotes the space of bounded linear isomorphisms. In particular, $B \in m \mathbb{R}_{\mu}\left(E_{1}, E_{0}\right)$ if and only if for every $\left(f, u_{0}\right) \in \mathbb{E}_{0}(J) \times \gamma \mathbb{E}_{1}$, there exists a unique solution $u \in \mathbb{E}_{1}(J)$ to the inhomogeneous Cauchy problem

$$
\left\{\begin{array}{l}
\dot{u}(t)+B u(t)=f(t), \quad t \in \dot{J}, \\
u(0)=u_{0} .
\end{array}\right.
$$

Moreover, in the current setting, it follows that $\gamma \mathbb{E}_{1} \doteq\left(E_{0}, E_{1}\right)_{\mu, \infty}^{0}$, i.e., the trace space $\gamma \mathbb{E}_{1}$ is topologically equivalent to the noted continuous interpolation spaces of Da Prato and Grisvard, cf. $[1,7,9,24]$.

\section{Well-posedness of $(2.4)$}

Well-posedness of the averaged mean curvature flow is well established in the literature, we reference the work of Escher and Simonett [14] for fundamental local well-posedness in arbitrary space dimensions with rough initial data, and we also reference Athanassenas [5], who considers rotationally symmetric surfaces in arbitrary space dimenions $\mathbb{R}^{n}$, satisfying Neumann boundary conditions. In the current periodic setting in $\mathbb{R}^{3}$, we establish the following well-posedness of (2.4).

THEOREM 3.1 Fix $\alpha \in(0,1)$ and take $\mu \in[1 / 2,1]$ so that $2 \mu+\alpha \notin \mathbb{Z}$. For every initial value $r_{0} \in V_{\mu}:=h^{2 \mu+\alpha}(\mathbb{T}) \cap[r>0]$, there exists a unique solution to (2.4),

$$
r\left(\cdot, r_{0}\right) \in C_{1-\mu}^{1}\left(J\left(r_{0}\right), h^{\alpha}(\mathbb{T})\right) \cap C_{1-\mu}\left(J\left(r_{0}\right), h^{2+\alpha}(\mathbb{T})\right),
$$

on the maximal interval of existence $J\left(r_{0}\right):=\left[0, t^{+}\left(r_{0}\right)\right)$. Further, we conclude that 
a. solutions have the additional regularity

$$
r\left(\cdot, r_{0}\right) \in C^{\omega}\left(\left(0, t^{+}\left(r_{0}\right)\right) \times \mathbb{T}\right) \quad \text { for all } \quad r_{0} \in V_{\mu} .
$$

b. (2.4) generates a real-analytic semiflow on $V_{\mu}$.

c. if there exists $0<M<\infty$ so that the bounds

- $r\left(t, r_{0}\right)(x) \geqslant 1 / M$, for all $x \in \mathbb{T}$, and

- $\left\|r\left(t, r_{0}\right)\right\|_{h^{\theta}} \leqslant M$, for some $\theta \in(1+\alpha, \infty) \backslash \mathbb{Z}$,

hold for all $t \in J\left(r_{0}\right)$, then $t^{+}\left(r_{0}\right)=\infty$.

To economize notation, we define the spaces, for $\alpha \in(0,1)$ fixed,

$$
E_{0}:=h^{\alpha}(\mathbb{T}), \quad E_{1}:=h^{2+\alpha}(\mathbb{T}), \quad \text { and } \quad E_{\mu}:=\left(E_{0}, E_{1}\right)_{\mu, \infty}^{0}, \quad \mu \in(0,1) .
$$

Utilizing well-known results regarding the continuous interpolation method $(\cdot, \cdot)_{\mu, \infty}^{0}$ and littleHölder spaces, we conclude

$$
E_{\mu}=h^{2 \mu+\alpha}(\mathbb{T}) \quad \text { (up to equivalent norms), for } 2 \mu+\alpha \notin \mathbb{Z},
$$

cf. [22, 24]. Further, let $V$ be the set of functions $r: \mathbb{T} \rightarrow \mathbb{R}$ such that $r(x)>0$ for all $x \in \mathbb{T}$ and define $V_{\mu}:=V \cap E_{\mu}$ for $\mu \in[0,1]$. Note that $V_{\mu}$ is an open subset of $E_{\mu}$ for all $\mu \in[0,1]$.

Before we prove the theorem, we reformulate the problem (2.4) in order to make explicit the quasilinear structure of the equation. In particular, we note that

$$
\begin{aligned}
G(r) & =-Q(r) r+f(r) \quad \text { where } \quad(Q, f): V_{1 / 2} \rightarrow \mathcal{L}\left(E_{1}, E_{0}\right) \times E_{0}, \\
Q(r) \rho & :=\frac{\sqrt{1+r_{x}^{2}}}{S(r)} \int_{\mathbb{T}} \frac{r(x) \rho_{x x}(x)}{\left(1+r_{x}^{2}(x)\right)} d x-\frac{\rho_{x x}}{\left(1+r_{x}^{2}\right)}, \\
f(r) & :=2 \pi \frac{\sqrt{1+r_{x}^{2}}}{S(r)}-\frac{1}{r}, \quad \rho \in E_{1}, r \in V_{1 / 2} .
\end{aligned}
$$

Lemma 3.2 Let $\mu \in[1 / 2,1]$. Then

$$
(Q, f) \in C^{\omega}\left(V_{\mu}, \mathfrak{m} R_{v}\left(E_{1}, E_{0}\right) \times E_{0}\right), \quad v \in(0,1] .
$$

Proof. The regularity of the mappings is standard from techniques in nonlinear analysis, noting that every $r \in V_{\mu}$ is strictly positive on $\mathbb{T}$ and $V_{\mu} \hookrightarrow h^{1+\alpha}(\mathbb{T})$ for $\mu \in[1 / 2,1]$. Hence, we focus on the maximal regularity result. In particular, for $r \in V_{\mu}$ fixed, it remains to show that $Q(r) \in M R_{v}\left(E_{1}, E_{0}\right), v \in(0,1]$.

Separating the terms of the operator $Q(r)$, it follows from [22, Theorem 5.2] that the secondorder uniformly elliptic operator with variable coefficients,

$$
-\frac{1}{\left(1+r_{x}^{2}\right)} \partial_{x}^{2}
$$

is in the maximal regularity class $m R_{v}\left(E_{1}, E_{0}\right), v \in(0,1]$. Meanwhile, we note that

$$
\begin{array}{r}
\left\|\frac{\sqrt{1+r_{x}^{2}}}{S(r)} \int_{\mathbb{T}} \frac{r(x)}{\left(1+r_{x}^{2}(x)\right)} \rho_{x x}(x) d x\right\|_{E_{0}} \leqslant C(r) \int_{\mathbb{T}}\left|\rho_{x x}(x)\right| d x \leqslant 2 \pi C(r)\|\rho\|_{C^{2}(\mathbb{T})} \\
\Longrightarrow \quad\left[\rho \mapsto \frac{\sqrt{1+r_{x}^{2}}}{S(r)} \int_{\mathbb{T}} \frac{r(x)}{\left(1+r_{x}^{2}(x)\right)} \rho_{x x}(x) d x\right] \in \mathcal{L}\left(h^{2+\beta}(\mathbb{T}), E_{0}\right),
\end{array}
$$


for any $0<\beta<\alpha, r \in V_{1 / 2}$, where $C(r)>0$ depends on the $h^{1+\alpha}(\mathbb{T})$-norm of $r$ and $\min _{x \in \mathbb{T}} r(x)$. Thus, from the interpolation inequality, in conjunction with the identity $h^{2+\beta}(\mathbb{T})=\left(E_{0}, E_{1}\right)_{\theta, \infty}^{0}$, $\theta=1-\frac{\alpha-\beta}{2}$, and an application of Young's inequality, we realize this term as a lower order perturbation, cf. [14, p. 2793]. The operator $Q(r)$ then satisfies maximal regularity by a well-known perturbation result, cf. [1, 14, 22].

Proof of Theorem 3.1. For $\mu \in[1 / 2,1)$, the main statement of the theorem, and parts (b) and (c), follow from the results [7] of Clément and Simonett regarding well-posedness of quasilinear problems, in the presence of sufficiently regular mappings $Q$ and $f$, and maximal regularity properties, as established in Lemma 3.2. Meanwhile, when $\mu=1$, we must consider (2.4) as a fully nonlinear problem, for which DaPrato and Grisvard [9] have established well-posedness and Angenent [3] has established semiflow properties in the presence of maximal regularity properties for the Fréchet derivatives $D G(\rho)$. Indeed, computing the Fréchet derivative $D G(\rho)$, one sees the structure

$$
D G(r) \rho=\frac{\rho_{x x}}{\left(1+r_{x}^{2}\right)}-\frac{\sqrt{1+r_{x}^{2}}}{S(r)} \int_{\mathbb{T}} \frac{\rho_{x x}}{\left(1+r_{x}^{2}\right)^{3 / 2}} d x+\mathbb{B}(r) \rho,
$$

for $\rho \in E_{1}$, where $\mathbb{B}(r)$ is a first-order differential operator. Hence, we apply the same perturbation technique as in the proof of Lemma 3.2 to establish maximal regularity properties for $D G(r), r \in$ $V_{1}$.

To prove the additional regularity of solutions, part $(a)$ of the theorem, we rely on a paramatertrick that goes back to Masuda [25] and Angenent [3, 4], where one can introduce parameters and apply the implicit function theorem to obtain regularity results for solutions, see also [12, 13, 23]. In particular, we define the translation operator $T_{a}: \mathbb{T} \rightarrow \mathbb{T}$ which takes $x \in \mathbb{T}$ to the unique element of $\mathbb{T}$ which resides in the coset $[x+a] \in \mathbb{R} / 2 \pi \mathbb{Z}$, for $a \in \mathbb{R}$. Given a solution $r\left(\cdot, r_{0}\right)$ to (2.4), for some $r_{0} \in V_{\mu}$, we take $t_{1} \in\left(0, t^{+}\left(r_{0}\right)\right)$ and consider the function

$$
r_{\lambda, a}(t, x):=r\left((1+\lambda) t, r_{0}\right)\left(T_{t a} x\right), \quad t \in I:=\left[0, t_{1}\right], x \in \mathbb{T},
$$

for $(\lambda, a) \in(-\delta, \delta)^{2}$, with $\delta>0$ chosen sufficiently small. It follows that $r_{\lambda, a} \in \mathbb{E}_{1}(I)$ and is a zero of the operator $\Phi:\left(\mathbb{E}_{1}(I) \cap C(I, V)\right) \times(-\delta, \delta)^{2} \rightarrow \mathbb{E}_{0}(I) \times E_{\mu}$,

$$
\Phi(v,(\lambda, a))=\left(\partial_{t} v-(1+\lambda) G(v)-a \partial_{x} v, \gamma v-r_{0}\right),
$$

where the spaces $\mathbb{E}_{j}(I)$ are defined as in (2.6) above. One quickly verifies that $\Phi$ is real-analytic on the domain specified and further, the Fréchet derivative of $\Phi$ (with respect to first variable v) evaluated at $r$ satisfies

$$
D_{1} \Phi(r,(0,0))=\left(\partial_{t}-D G(r), \gamma\right) \in \mathcal{L}_{i s o m}\left(\mathbb{E}_{1}(I), \mathbb{E}_{0}(I) \times E_{\mu}\right)
$$

by way of maximal regularity, cf. [7, Theorem 6.1]. The result now follows by an application of the implicit function theorem, cf. [23, Theorem 2.4].

\section{General properties of (2.4)}

We move on to investigate geometric properties of solutions. To begin, we verify that two important features of the averaged mean curvature flow hold for solutions of (2.4), namely volume-preservation and surface-area reduction. Following these verifications, we characterize the collection of equilibria to (2.4), which will be the central focus of the remainder of the article. 


\subsection{Volume-preservation and surface-area-reduction}

Suppose that $r \in C_{1-\mu}^{1}\left(J\left(r_{0}\right), E_{0}\right) \cap C_{1-\mu}\left(J\left(r_{0}\right), E_{1}\right)$ is a solution to (2.4) for some initial value $r_{0} \in V_{\mu}, \mu \in[1 / 2,1]$. Looking at the evolution of the surface-area functional $S$, making judicious use of integration by parts and periodicity to cancel boundary terms, we see that

$$
\begin{aligned}
\frac{d}{d t} S(r) & =\int_{\mathbb{T}}\left(\sqrt{1+r_{x}^{2}(x)}+\frac{r(x) r_{x}(x)}{\sqrt{1+r_{x}^{2}(x)}} \partial_{x}\right) G(r) d x \\
& =\int_{\mathbb{T}} \mathcal{H}(r)(x)[h(r)-\mathcal{H}(r)(x)] r(x) \sqrt{1+r_{x}^{2}(x)} d x \\
& =-\int_{\mathbb{T}}[h(r)-\mathcal{H}(r)(x)]^{2} r(x) \sqrt{1+r_{x}^{2}(x)} d x,
\end{aligned}
$$

where the last equation follows from

$$
\begin{array}{rl}
\int_{\mathbb{T}} & h(r)[h(r)-\mathcal{H}(r)(x)] r(x) \sqrt{1+r_{x}^{2}(x)} d x \\
= & {[h(r)]^{2}\left(\int_{\mathbb{T}} r(x) \sqrt{1+r_{x}^{2}(x)} d x\right)-h(r)\left(\int_{\mathbb{T}} \mathcal{H}(r)(x) r(x) \sqrt{1+r_{x}^{2}(x)} d x\right)} \\
= & S(r)[h(r)]^{2}-S(r)[h(r)]^{2}=0 .
\end{array}
$$

Thus, it follows that the surface area of the induced surfaces of revolution $\Gamma(r(t))$ is decreasing in $t$. In fact, we can see that the surface area is constant if and only if the mean curvature is constant, which coincides with an equilibrium surface. Meanwhile, looking at the evolution of the volume functional

$$
F(r):=\int_{\mathbb{T}} r^{2}(x) d x
$$

we see that

$$
\frac{d}{d t} F(r)=2 \int_{\mathbb{T}} r(x) G(r)(x) d x=2 \int_{\mathbb{T}} r(x) \sqrt{1+r_{x}^{2}(x)}(h(r)-\mathcal{H}(r)(x)) d x=0
$$

Therefore, taking into account the regularity of the operator $F$ in the topology of $E_{\mu}$, it follows that $F(r(t))=F\left(r_{0}\right)$ for all $t \in J\left(r_{0}\right)$.

\subsection{Characterization of equilibria}

Considering the equilibria, i.e. the steady states, of the problem (2.4), it follows immediately that $\bar{r} \in E_{\mu}$ is an equilibrium if and only if $G(\bar{r})=0$. Moreover, from the structure of the equation we conclude $G(\bar{r})=0$ if and only if $\mathcal{H}(\bar{r}) \equiv h(\bar{r})$, i.e. the mean curvature of the surface of revolution $\Gamma(\bar{r})$ must be constant on $\mathbb{T}$. Using results of Delaunay [11] and Kenmotsu [20] regarding surfaces of revolution with constant mean curvature, we characterize all equilibria of (2.4) as follows.

THEOREM 4.1 [Characterization of Equilibria] Fix $\alpha \in(0,1)$. Then $\bar{r} \in h^{2+\alpha}(\mathbb{T})$ is an equilibrium of the flow (2.4) if and only if $\bar{r}$ is a $2 \pi$-periodic undulary curve. Moreover, up to a translation along the axis of rotation, $\bar{r}$ can be expressed explicitly by the parametric equation (with respect to the 

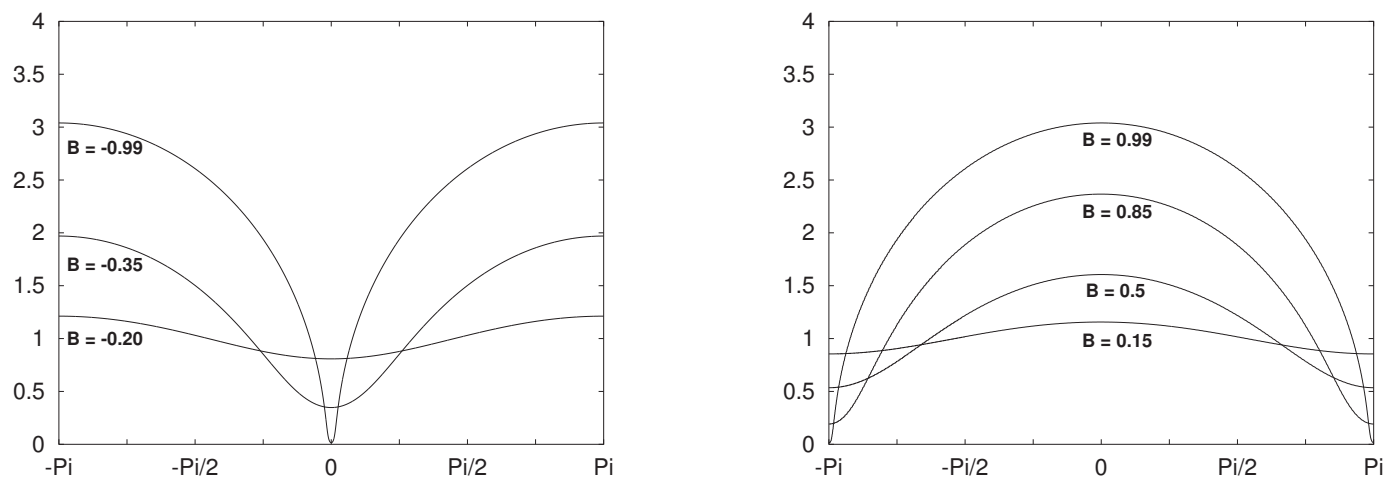

FIG. 1. Families of $2 \pi$ periodic undulary curves (with $k=1$ ) with values between $B=-.99$ and $B=0.99$, as indicated.

arclength parameter s),

$$
R(s ; \mathcal{H}, B):=\left(\int_{\pi / 2 \mathcal{H}}^{s} \frac{1+B \sin (\mathcal{H} t)}{\sqrt{1+B^{2}+2 B \sin (\mathcal{H} t)}} d t, \frac{\sqrt{1+B^{2}+2 B \sin (\mathcal{H} s)}}{|\mathcal{H}|}\right),
$$

for constants $\mathcal{H}>0$ and $B \in(-1,1)$ which satisfy the relationship

$$
\frac{\pi \mathcal{H}}{k}=\int_{\pi / 2}^{3 \pi / 2} \frac{1+B \sin t}{\sqrt{1+B^{2}+2 B \sin t}} d t, \text { for some } k \in \mathbb{N} .
$$

Proof. Delaunay [11] proved that every closed surface of revolution in $\mathbb{R}^{3}$ with constant mean curvature is either a sphere, a catenoid, a nodoid or an unduloid. Looking at the associated profile curves, the only surfaces which fit into our current setting (taking into account periodicity, embeddedness and regularity constraints) are the family of $2 \pi$-periodic unduloids, for which Kenmotsu [20] derived an explicit parametrization of profile curves. The parametrization presented herein is a slightly modified version of Kenmotsu's formula, where we have adjusted the graph to be symmetric about the $y$-axis and to exhibit $\frac{2 \pi}{k}$-periodicity in the $x$-variable, for a given value $k \in \mathbb{N}$.

To stimulate the reader's curiosity, we include in Fig. 1 above some graphs of undulary curves $\bar{r}$ which, by the previous theorem, are the only equilibria of (2.4) (up to shifts along the $x$-axis).

\section{Stability behavior of cylinders}

For the remainder of the paper, we equate the constant function $r_{\star}(x) \equiv r_{\star}>0$ with the cylinder $\Gamma\left(r_{\star}\right)$ with radius $r_{\star}$ and length $2 \pi$. The stability of cylinders under the averaged mean curvature flow is a particularly vibrant question, in rotationally symmetric settings. Considering $n$-dimensional smooth surfaces of revolution with Neumann boundary conditions, Athanassenas [5] showed that initial surfaces satisfying a particular perimetric-type inequality, comparing the surface area, enclosed volume, and length of the interval of revolution, will have global solutions which eventually converge to an $n$-dimensional cylinder. More recent results by Hartley [17] indicate that one may be able to generalize this convergence result to rough initial data without 
requiring rotational symmetry of initial data, though we again see a perimetric-type inequality in his results. Athanassenas notes in [5] that the volume constraint imposed by the perimetric-type inequality eliminates the possibility of unduloid equilibria, in the absence of which one can conclude convergence to cylinders. However, in the current paper, we are interested precisely in the family of unduloids and how they interact (bifurcate from) the family of cylinders, for which a finer analysis is necessary.

In this section, we will demonstrate that the family of cylinders with radius $r_{\star}>1$ is exponentially (nonlinearly) stable, cf. Theorem 5.3. Moreover, we establish instability of cylinders with radius $0<r_{\star}<1$ and develop a setting within which dynamical systems techniques become accessible. In the next section, we will show how this setting can be applied to study the bifurcation which occurs at the critical radius $r_{\star}=1$, and subsequent bifurcations at smaller radii, cf. Theorem 6.1.

We first note that functions in the little-Hölder spaces, $r \in h^{\sigma}(\mathbb{T})$, obey the Fourier series representation (cf. [22])

$$
r(x)=\sum_{k \in \mathbb{Z}} \hat{r}(k) e^{i k x}, \quad x \in \mathbb{T} ; \quad \text { where } \hat{r}(k):=\frac{1}{2 \pi} \int_{\mathbb{T}} r(x) e^{-i k x} d x
$$

We then compute the linearization

$$
\begin{aligned}
D G\left(r_{\star}\right) \rho & =-D \mathcal{H}\left(r_{\star}\right) \rho+\frac{1}{2 \pi} \int_{\mathbb{T}}\left[D \mathcal{H}\left(r_{\star}\right) \rho\right](x) d x, \quad \rho \in h^{2+\alpha}(\mathbb{T}) \\
& =\left(\rho r_{\star}^{-2}+\rho_{x x}\right)-\frac{1}{2 \pi} \int_{\mathbb{T}}\left(\rho(x) r_{\star}^{-2}+\rho_{x x}(x)\right) d x
\end{aligned}
$$

and realize it in the form of the Fourier multiplier

$$
\left[D G\left(r_{\star}\right) \rho\right](x)=\sum_{k \in \mathbb{Z} \backslash\{0\}}\left(r_{\star}^{-2}-k^{2}\right) \hat{\rho}(k) e^{i k x}, \quad \rho \in h^{2+\alpha}(\mathbb{T}), \quad x \in \mathbb{T} .
$$

From this expression, it is easy to verify that the point spectrum of $D G\left(r_{\star}\right)-$ which must in fact coincide with the entire spectrum $\sigma\left(D G\left(r_{\star}\right)\right)$ due to compactness of the embedding $h^{2+\alpha}(\mathbb{T}) \hookrightarrow$ $h^{\alpha}(\mathbb{T})-$ is

$$
\sigma\left(D G\left(r_{\star}\right)\right)=\{0\} \cup\left\{\left(r_{\star}^{-2}-k^{2}\right): k \in \mathbb{Z} \backslash\{0\}\right\} .
$$

Notice that, for $r_{\star}>1$, this spectrum is contained in the left-half of the complex plane, which we can take advantage of in order to establish stability of the equilibrium $r_{\star}$. However, the presence of 0 in the spectrum is troublesome, as it indicates the presence of a center manifold which disturbs, but does not derail, our stability argument. To get around this hurdle, we proceed to reinterpret the operator $G$, and subsequently $D G$, in a reduced setting where this eigenvalue no longer shows up. The following technique takes advantage of the volume-preserving nature of (2.4) in order to investigate local properties of the problem, it is motivated by methods of Prokert [27] and Vondenhoff [30] and employed by the author in [23]. 


\subsection{Zero-mean functions and the reduced problem}

For this section, we consider arbitrary constants $r_{\star}>0$ and $\sigma \in \mathbb{R}_{+} \backslash \mathbb{Z}$, unless otherwise stated. We first introduce the mappings

$$
P_{0} r:=r-\frac{1}{2 \pi} \int_{\mathbb{T}} r(x) d x, \quad \text { and } \quad Q_{0}:=1-P_{0}
$$

which define projections on $h^{\sigma}(\mathbb{T})$. Denote by $h_{0}^{\sigma}(\mathbb{T})$ the image $P_{0}\left(h^{\sigma}(\mathbb{T})\right)$, which coincides with the zero-mean functions on $\mathbb{T}$ in the regularity class $h^{\sigma}(\mathbb{T})$. We then have the topological decomposition

$$
h^{\sigma}(\mathbb{T})=h_{0}^{\sigma}(\mathbb{T}) \oplus Q_{0}\left(h^{\sigma}(\mathbb{T})\right) \cong h_{0}^{\sigma}(\mathbb{T}) \oplus \mathbb{R} .
$$

In the sequel, we equate the constant function $[\eta(x) \equiv \eta] \in Q_{0}\left(h^{\sigma}(\mathbb{T})\right)$ with the value $\eta \in \mathbb{R}$, and we denote each simply as $\eta$.

Consider the operator

$$
\Phi(r, \tilde{r}, \eta):=\left(P_{0} r-\tilde{r}, F(r)-F(\eta)\right), \quad \text { with } \quad F(r):=\int_{\mathbb{T}} r^{2}(x) d x,
$$

which is a real-analytic operator from $h^{\sigma}(\mathbb{T}) \times h_{0}^{\sigma}(\mathbb{T}) \times \mathbb{R}$ into $h_{0}^{\sigma}(\mathbb{T}) \times \mathbb{R}$. Notice that $\Phi\left(r_{\star}, 0, r_{\star}\right)=$ $(0,0)$ and

$$
\begin{aligned}
D_{1} \Phi\left(r_{\star}, 0, r_{\star}\right) & =\left(P_{0}, 2 \int_{\mathbb{T}} r_{\star} i d_{h^{\sigma}(\mathbb{T})} d x\right) \\
& =\left(P_{0}, 4 \pi r_{\star} Q_{0}\right) \in \mathcal{L}_{i s o m}\left(h^{\sigma}(\mathbb{T}), h_{0}^{\sigma}(\mathbb{T}) \times \mathbb{R}\right),
\end{aligned}
$$

i.e. the Fréchet derivative of $\Phi$ with respect to the first variable, at $\left(r_{\star}, 0, r_{\star}\right)$, is a bounded linear isomorphism. Hence, it follows from the implicit function theorem, cf. [10, Theorem 15.3], that there exist neighborhoods

$$
\left(0, r_{\star}\right) \in U=U_{0} \times U_{1} \subset h_{0}^{\sigma}(\mathbb{T}) \times \mathbb{R}, \quad r_{\star} \in U_{2} \subset h^{\sigma}(\mathbb{T}),
$$

and a $C^{\omega}$ function $\psi_{\star}: U \rightarrow U_{2}$ such that, for all $(r, \tilde{r}, \eta) \in U_{2} \times U$,

$$
\Phi(r, \tilde{r}, \eta)=(0,0) \text { if and only if } r=\psi_{\star}(\tilde{r}, \eta) .
$$

Note that the quantity $F(r)$ corresponds to the volume over $\mathbb{T}$ enclosed by the surface $\Gamma(r)$ (modulo a factor of $2 \pi$ ), which is a preserved quantity under (2.4), as discussed in Subsection 4.1. Then, we can interpret $\psi_{\star}(\cdot, \eta)$ as a lifting operator which takes a zero-mean function $\tilde{r} \in h_{0}^{\sigma}(\mathbb{T})$ to an associated function in the equivolume set

$$
m_{\eta}^{\sigma}:=\left\{r \in h^{\sigma}(\mathbb{T}): F(r)=F(\eta)\right\},
$$

which contains all the profile functions corresponding to surfaces enclosing the same volume as the cylinder $\eta$. In particular, if we choose $\eta$ so that $F\left(r_{0}\right)=F(\eta)$, it follows from section 4.1 that $r\left(t, r_{0}\right) \in m_{\eta}^{\alpha}$ for all $t \in J\left(r_{0}\right)$.

REMARK 5.1 For $r_{\star}>0$ fixed, we can immediately state the following properties of $\psi_{\star}$ which come from the definition and elucidate the relationship between $P_{0}$ and $\psi_{\star}$. 
a. $P_{0} \psi_{\star}(\tilde{r}, \eta)=\tilde{r}$ for all $(\tilde{r}, \eta) \in U$.

b. Given $r \in \psi_{\star}(U) \cap m_{\eta}^{\sigma}$, it follows that $\psi_{\star}\left(P_{0} r, \eta\right)=r$.

c. $\psi_{\star}(0, \eta)=\eta$, for $\eta \in U_{1}$. This and the preceding remark follow from the fact that $F(\eta)$ is injective as a function on $(0, \infty)$.

d. It follows from the identity $\Phi\left(\psi_{\star}(\tilde{r}, \eta), \tilde{r}, \eta\right)=(0,0)$ and differentiating with respect to $\tilde{r}$ that

$$
D_{1} \psi_{\star}(0, \eta) h=h, \quad h \in h_{0}^{\sigma}(\mathbb{T}), \eta \in U_{1} .
$$

e. Note $\psi_{\star}\left(U_{0}, \eta\right) \subset m_{\eta}^{\sigma}$ for $\eta \in U_{1}$ and we have the representation

$$
\psi_{\star}(\tilde{r}, \eta)=\left(P_{0}+Q_{0}\right) \psi_{\star}(\tilde{r}, \eta)=\tilde{r}+\frac{1}{2 \pi} \int_{\mathbb{T}} \psi_{\star}(\tilde{r}, \eta)(x) d x, \quad \tilde{r} \in U_{0} .
$$

Hence, we see that $m_{\eta}^{\sigma} \cap U_{2}$ is the graph of a $\mathbb{R}$-valued function over $h_{0}^{\sigma}(\mathbb{T})$ which is therefore a Banach manifold, with real-analytic local parametrization $\psi_{\star}(\cdot, \eta)$.

f. A priori, $\psi_{\star}(\cdot, \eta)$ depends upon the parameter $\sigma$, however it follows from the preceding representation that

$$
\psi_{\star}(\cdot, \eta): U_{0} \cap h_{0}^{\tilde{\sigma}}(\mathbb{T}) \rightarrow h^{\tilde{\sigma}}(\mathbb{T}), \quad \tilde{\sigma} \in \mathbb{R}_{+} \backslash \mathbb{Z},
$$

so that $\psi_{\star}$ preserves the spacial regularity of functions regardless of the regularity parameter $\sigma$ with which $\psi_{\star}$ was constructed. However, notice that the neighborhood $U_{0}$ will remain intrinsically linked with the parameter which was used to construct $\psi_{\star}$.

Fix $r_{\star}>0$ and define the reduced governing operator

$$
\mathcal{G}(\tilde{r}, \eta):=P_{0} G\left(\psi_{\star}(\tilde{r}, \eta)\right)
$$

which acts on $(\tilde{r}, \eta)$ in a neighborhood $U$, as constructed for $\psi_{\star}$ above. We need to be careful with the regularity of the zero-mean functions $\tilde{r}$ that we plug into $\mathcal{G}$, so we will assume throughout that $\alpha \in(0,1)$ is given and we define the lifting $\psi_{\star}$, and the related neighborhood $U=U_{0} \times U_{1}$, within the setting of $h_{0}^{\alpha}(\mathbb{T})$. We then take $\mathcal{G}(\cdot, \eta)$ to be acting on the functions $\tilde{r} \in U_{0} \cap h_{0}^{2+\alpha}(\mathbb{T}), \eta \in U_{1}$. With this reduced operator, we define the $\eta$-dependent reduced problem

$$
\begin{cases}\tilde{r}_{t}(t, x)=G(\tilde{r}(t, x), \eta), & t>0, x \in \mathbb{T}, \\ \tilde{r}(0, x)=\left[P_{0} r_{0}\right](x), & x \in \mathbb{T} .\end{cases}
$$

Theoretically, we can choose the parameter $\eta \in U_{1}$ arbitrarily, however, in practice, we will choose $\eta$ for which $F(\eta)=F\left(r_{0}\right)$, which essentially gives us the freedom to consider non-volume preserving perturbations $r_{0}$ of the cylinder $r_{\star}$ in our stability analysis.

Fix $\alpha \in(0,1)$ and we denote the spaces

$$
F_{0}:=h_{0}^{\alpha}(\mathbb{T}), \quad F_{1}:=h_{0}^{2+\alpha}(\mathbb{T}), \quad \text { and } \quad F_{\mu}:=\left(F_{0}, F_{1}\right)_{\mu, \infty}^{0}, \quad \mu \in(0,1),
$$

so that $F_{\mu}=P_{0} E_{\mu}$ for $\mu \in[0,1]$. Moreover, for $\mu \in(0,1]$ and closed intervals $J \subseteq \mathbb{R}_{+}$with $0 \in J$, define the spaces

$$
\begin{aligned}
& \mathbb{E}_{0}(J):=B U C_{1-\mu}\left(J, E_{0}\right), \\
& \mathbb{E}_{1}(J):=B U C_{1-\mu}^{1}\left(J, E_{0}\right) \cap B U C_{1-\mu}\left(J, E_{1}\right),
\end{aligned}
$$


and

$$
\begin{aligned}
& \mathbb{F}_{0}(J):=B U C_{1-\mu}\left(J, F_{0}\right), \\
& \mathbb{F}_{1}(J):=B U C_{1-\mu}^{1}\left(J, F_{0}\right) \cap B U C_{1-\mu}\left(J, F_{1}\right),
\end{aligned}
$$

within which we will discuss solutions to (2.4) and the reduced problem (5.7), respectively. We can immediately see how the lifting operator $\psi_{\star}$ connects these two problems.

LEMma 5.2 Fix $r_{\star}>0, \eta \in U_{1}$ and $J:=[0, T]$ for $T>0$. Then

$$
\psi_{\star}(\cdot, \eta): \mathbb{F}_{1}(J) \cap C\left(J, U_{0}\right) \longrightarrow \mathbb{E}_{1}(J), \quad \text { with } \quad \psi_{\star}(\tilde{r}, \eta)(t):=\psi_{\star}(\tilde{r}(t), \eta) .
$$

Moreover, if $\tilde{r}_{0}:=P_{0} r_{0} \in F_{\mu}$ and $\tilde{r}=\tilde{r}\left(\cdot, \tilde{r}_{0}\right) \in \mathbb{F}_{1}(J) \cap C\left(J, U_{0}\right)$ is a solution to (5.7), for some $\mu \in[1 / 2,1]$, then $r:=\psi_{\star}(\tilde{r}, \eta)$ is the unique solution on the interval $J$ to $(2.4)$, with initial data $r_{0}=\psi_{\star}\left(\tilde{r}_{0}, \eta\right) \in E_{\mu}$.

Proof. Because of the local nature of the operator $\psi_{\star}$, we can assume, without loss of generality, that the neighborhood $U=U_{0} \times U_{1}$ is chosen sufficiently small to ensure that $\psi_{\star}$ is in the regularity class $C^{\omega}$ and the bounds

$$
\|\psi(\tilde{r}, \eta)\|_{E_{0}} \leqslant N \quad \text { and } \quad\left\|D_{1} \psi(\tilde{r}, \eta)\right\|_{\mathcal{L}\left(F_{0}, E_{0}\right)} \leqslant N
$$

hold uniformly for $(\tilde{r}, \eta) \in U$. Further, the embeddings

$$
\mathbb{F}_{1}(J) \hookrightarrow B U C\left(J, F_{\mu}\right) \hookrightarrow B U C\left(J, F_{0}\right), \quad \mu \in[1 / 2,1],
$$

follow from [1, Theorem III.2.3.3] and the continuous embedding of little-Hölder spaces, respectively. It is then straightforward, utilizing the representation for $\psi_{\star}$ given in Remarks 5.1(e) and these bounds, to verify (5.8).

Meanwhile, by (5.10) we have $r_{0}:=\psi_{\star}\left(\tilde{r}_{0}, \eta\right) \in V_{\mu}$, and so it follows from Theorem 3.1 that there exists a unique maximal solution

$$
r\left(\cdot, r_{0}\right) \in C_{1-\mu}^{1}\left(J\left(r_{0}\right), E_{0}\right) \cap C_{1-\mu}\left(J\left(r_{0}\right), E_{1}\right)
$$

to (2.4). Define $\rho(\cdot):=\psi(\tilde{r}(\cdot), \eta)$ as indicated and it suffices to show that $\rho_{t}(t)=G(\rho(t))$ for $t \in \dot{J}:=(0, T]$, since this will imply that $\rho(t)=r\left(t, r_{0}\right)$ by uniqueness and maximality of the solution $r\left(\cdot, r_{0}\right)$. So, let $t \in \dot{J}$ and consider the auxiliary problem

$$
\left\{\begin{array}{l}
\dot{\gamma}(\tau)=G(\gamma(\tau)), \quad \text { for } \tau \in[0, \varepsilon], \\
\gamma(0)=\rho(t),
\end{array}\right.
$$

which has a unique solution $\gamma \in C^{1}\left([0, \varepsilon], E_{0}\right) \cap C\left([0, \varepsilon], E_{1}\right)$ by Theorem 3.1, provided we choose $\varepsilon>0$ sufficiently small for the particular value $\rho(t) \in E_{1}$. By Remarks 5.1 and preservation of volume for solutions of (2.4), we have the representation $\gamma(\tau)=\psi_{\star}\left(P_{0} \gamma(\tau), \eta\right), \tau \in[0, \varepsilon]$. We then conclude the proof by computing

$$
\begin{aligned}
G(\rho(t)) & =\dot{\gamma}(0)=\left.\partial_{\tau}\left(\psi_{\star}\left(P_{0} \gamma(\tau), \eta\right)\right)\right|_{\tau=0}=D_{1} \psi_{\star}\left(P_{0} \gamma(0), \eta\right) P_{0} \dot{\gamma}(0) \\
& =D_{1} \psi_{\star}\left(P_{0} \rho(t), \eta\right) P_{0} G(\rho(t))=D_{1} \psi_{\star}(\tilde{r}(t), \eta) G(\tilde{r}(t), \eta) \\
& =\partial_{t}\left(\psi_{\star}(\tilde{r}(t), \eta)\right)=\rho_{t}(t) .
\end{aligned}
$$


Considering the stability of $r_{\star}$, we compute the linearization

$$
D_{1} G(\tilde{r}, \eta)=P_{0} D G\left(\psi_{\star}(\tilde{r}, \eta)\right) D_{1} \psi_{\star}(\tilde{r}, \eta), \quad(\tilde{r}, \eta) \in U,
$$

which simplifies at $\tilde{r}=0$, using (5.1) and Remarks 5.1(d),

$$
D_{1} G(0, \eta) \tilde{\rho}=P_{0}(D G(\eta)) D_{1} \psi(0, \eta) \tilde{\rho}=-P_{0}\left(P_{0} D \mathcal{H}(\eta)\right) \tilde{\rho}=-P_{0} D \mathcal{H}(\eta) \tilde{\rho}
$$

for $\tilde{\rho} \in h_{0}^{2+\alpha}(\mathbb{T})$. Recalling (5.3), and the reduced domain of definition for $\mathcal{G}$, we conclude

$$
\sigma\left(D_{1} \mathrm{G}(0, \eta)\right)=\left\{\left(\eta^{-2}-k^{2}\right): k \in \mathbb{Z} \backslash\{0\}\right\} .
$$

\subsection{Stability of cylinders with $r_{\star}>1$}

Define the exponentially weighted maximal regularity spaces

$$
\mathbb{F}_{j}\left(\mathbb{R}_{+}, \omega\right):=\left\{f:(0, \infty) \rightarrow F_{0} \mid\left[t \mapsto e^{\omega t} f(t)\right] \in \mathbb{F}_{j}\left(\mathbb{R}_{+}\right)\right\}, \quad \omega \in \mathbb{R}, j=0,1,
$$

which are Banach spaces when equipped with norms $\|u\|_{\mathbb{F}_{j}\left(\mathbb{R}_{+}, \omega\right)}:=\left\|e^{\omega t} u\right\|_{\mathbb{F}_{j}\left(\mathbb{R}_{+}\right)}$. We use these spaces in order to show the following stability result, which essentially tells us that $h^{2 \mu+\alpha}$-small perturbations of the cylinder $r_{\star}>1$ will have global solutions which converge exponentially fast to a cylinder $\eta$, which is close to $r_{\star}$.

Theorem 5.3 Fix $\alpha \in(0,1), \mu \in[1 / 2,1]$ so that $2 \mu+\alpha \notin \mathbb{Z}$, and let $r_{\star}>1$. There exist positive constants $\varepsilon=\varepsilon\left(r_{\star}\right), \delta=\delta\left(r_{\star}\right)$ and $\omega=\omega\left(r_{\star}, \delta\right)$, such that problem (2.4) with initial data $r_{0} \in \mathbb{B}_{h^{2 \mu+\alpha}}\left(r_{\star}, \varepsilon\right)$ has a unique global solution

$$
r\left(\cdot, r_{0}\right) \in C_{1-\mu}^{1}\left(\mathbb{R}_{+}, h^{\alpha}(\mathbb{T})\right) \cap C_{1-\mu}\left(\mathbb{R}_{+}, h^{2+\alpha}(\mathbb{T})\right),
$$

and there exist $\eta=\eta\left(r_{0}\right) \in\left(r_{\star}-\delta, r_{\star}+\delta\right)$ and $M=M(\alpha)>0$ for which the bound

$$
t^{1-\mu}\left\|r\left(t, r_{0}\right)-\eta\right\|_{h^{2+\alpha}}+\left\|r\left(t, r_{0}\right)-\eta\right\|_{h^{2 \mu+\alpha}} \leqslant M e^{-\omega t}\left\|r_{0}-r_{\star}\right\|_{h^{2 \mu+\alpha}}
$$

holds uniformly for $t \geqslant 0$.

Proof. We demonstrate this result by first showing that 0 is exponentially stable in the reduced problem (5.7), and then we lift solutions using $\psi_{\star}$ and show that exponential convergence is preserved in the lifting process.

(i) Fix $\delta \in\left(0, r_{\star}-1\right)$. Notice that the linearization of $\mathcal{G}(0, \eta)$ has the structure

$$
D_{1} G(0, \eta) \tilde{\rho}=P_{0}\left(\tilde{\rho}_{x x}+\eta^{-2} \tilde{\rho}\right)=\left(\tilde{\rho}_{x x}+\eta^{-2} \tilde{\rho}\right)-\frac{1}{2 \pi} \int_{\mathbb{T}}\left(\tilde{\rho}_{x x}(x)+\eta^{-2} \tilde{\rho}(x)\right) d x,
$$

from which, similar to the argument presented in Theorem 3.1, we realize $D_{1} g(0, \eta)$ as a lower order perturbation of the second order derivative operator $\partial_{x}^{2}$. Hence, it follows that $D_{1} g(0, \eta) \in$ $\mathcal{H}\left(F_{1}, F_{0}\right)$; the class of infinitesimal generators of analytic semigroups on $F_{0}$ with domain $F_{1}$. Moreover, it follows from (5.13) that the spectral type

$$
\text { type }\left(D_{1} g(0, \eta)\right)<\frac{1-\left(r_{\star}-\delta\right)^{2}}{\left(r_{\star}-\delta\right)^{2}}<0, \quad \text { for all } \quad \eta \in\left(r_{\star}-\delta, r_{\star}+\delta\right) \text {. }
$$


Hence, if we choose $\omega \in\left(0, \frac{\left(r_{\star}-\delta\right)^{2}-1}{\left(r_{\star}-\delta\right)^{2}}\right)$, then it follows by [1, Theorem III.3.4.1 and Remarks 3.4.2(b)] that the exponentially weighted spaces ,

$$
\left(\mathbb{F}_{0}\left(\mathbb{R}_{+}, \omega\right), \mathbb{F}_{1}\left(\mathbb{R}_{+}, \omega\right)\right),
$$

are a pair of maximal regularity for $D_{1} G(0, \eta)$, for all $\eta \in\left(r_{\star}-\delta, r_{\star}+\delta\right)$.

Define the operator

$$
\mathcal{K}\left(\tilde{r}, \tilde{r}_{0}, \eta\right):=\left(\partial_{t} \tilde{r}-\mathcal{G}(\tilde{r}, \eta), \gamma \tilde{r}-\tilde{r}_{0}\right),
$$

acting on $\mathbb{U}:=\left(\mathbb{F}_{1}\left(\mathbb{R}_{+}, \omega\right) \cap C\left(\mathbb{R}_{+}, U_{0}\right)\right) \times\left(U_{0} \cap F_{\mu}\right) \times U_{1}$, which is an open subset of $\mathbb{F}_{1}\left(\mathbb{R}_{+}, \omega\right) \times$ $F_{\mu} \times \mathbb{R}$. It follows that

$$
\mathcal{K} \in C^{\omega}\left(\mathbb{U}, \mathbb{F}_{0}\left(\mathbb{R}_{+}, \omega\right) \times F_{\mu}\right),
$$

by analyzing the mapping properties of the individual operators $\gamma, \partial_{t}$ and 9 on their associated domains of definition. The fact that $\mathcal{G}(\cdot, \eta)$ maps $\left(\mathbb{F}_{1}\left(\mathbb{R}_{+}, \omega\right) \cap C\left(\mathbb{R}_{+}, U_{0}\right)\right)$ into $\mathbb{F}_{0}\left(\mathbb{R}_{+}, \omega\right)$ follows by utilizing the representation (3.2), and bounding individual terms of the resulting expression using the embeddings (5.10) and the exponential boundedness of functions in $\mathbb{F}_{1}\left(\mathbb{R}_{+}, \omega\right)$.

Meanwhile, notice that $\mathcal{K}(0,0, \eta)=(0,0)$ and

$$
D_{1} \mathcal{K}(0,0, \eta)=\left(\partial_{t}-D_{1} G(0, \eta), \gamma\right) \in \mathcal{L}_{\text {isom }}\left(\mathbb{F}_{1}\left(\mathbb{R}_{+}, \omega\right), \mathbb{F}_{0}\left(\mathbb{R}_{+}, \omega\right) \times F_{\mu}\right),
$$

for $\eta \in\left(r_{\star}-\delta, r_{\star}+\delta\right)$. Thus, by the implicit function theorem there exists an open neighborhood $\left(0, r_{\star}\right) \in \tilde{U} \subset F_{\mu} \times \mathbb{R}$ and a $C^{\omega}$ mapping $\kappa: \tilde{U} \rightarrow \mathbb{F}_{1}\left(\mathbb{R}_{+}, \omega\right)$ such that

$$
\mathcal{K}\left(\kappa\left(\tilde{r}_{0}, \eta\right), \tilde{r}_{0}, \eta\right)=(0,0) \text { for all }\left(\tilde{r}_{0}, \eta\right) \in \tilde{U} .
$$

In particular, $\kappa\left(\tilde{r}_{0}, \eta\right)$ is a global solution to (5.7) with parameter $\eta$ and initial data $\tilde{r}_{0} \in F_{\mu}$, which converges exponentially fast to 0 . Without loss of generality, we assume $\tilde{U} \subset U_{1} \times\left(r_{\star}-\delta, r_{\star}+\delta\right)$.

(ii) Choose $\varepsilon>0$ so that, for every $r_{0} \in \mathbb{B}_{E_{\mu}}\left(r_{\star}, \varepsilon\right)$, there exists $(\tilde{r}, \eta) \in \tilde{U}$ for which $\Phi\left(r_{0}, \tilde{r}, \eta\right)=(0,0)$. Let $r_{0} \in \mathbb{B}_{E_{\mu}}\left(r_{\star}, \varepsilon\right)$, fix $\eta \in\left(r_{\star}-\delta, r_{\star}+\delta\right)$ so that $F\left(r_{0}\right)=F(\eta)$ and define

$$
r(\cdot):=\psi_{\star}\left(\kappa\left(P_{0} r_{0}, \eta\right), \eta\right) .
$$

Using Lemma 5.2, one verifies that $r \in C_{1-\mu}^{1}\left(\mathbb{R}_{+}, h^{\alpha}(\mathbb{T})\right) \cap C_{1-\mu}\left(\mathbb{R}_{+}, h^{2+\alpha}(\mathbb{T})\right)$ is the unique global solution to (2.4) with initial data $r_{0}$. Hence, it remains to show exponential convergence of $r$ to the cylinder $\eta$. Noting that $\kappa(0, \eta) \equiv 0$, and utilizing Remarks 5.1 and the mean value theorem, we compute

$$
\begin{aligned}
r(t)-\eta & =\left(P_{0}+Q_{0}\right)\left(\psi_{\star}\left(\kappa\left(P_{0} r_{0}, \eta\right)(t), \eta\right)-\psi_{\star}(\kappa(0, \eta)(t), \eta)\right) \\
& =\kappa\left(P_{0} r_{0}, \eta\right)(t)+Q_{0}\left(\psi_{\star}\left(\kappa\left(P_{0} r_{0}, \eta\right)(t), \eta\right)-\psi_{\star}(\kappa(0, \eta)(t), \eta)\right) \\
& =\kappa\left(P_{0} r_{0}, \eta\right)(t)+\frac{1}{2 \pi} \int_{\mathbb{T}} \int_{0}^{1} D_{1} \psi_{\star}\left(\tau \kappa\left(P_{0} r_{0}, \eta\right)(t), \eta\right) \kappa\left(P_{0} r_{0}, \eta\right)(t, x) d \tau d x,
\end{aligned}
$$

for $t>0$. We bound the terms

$$
e^{\omega t} t^{1-\mu}\left\|\kappa\left(P_{0} r_{0}, \eta\right)(t)\right\|_{F_{0}} \quad \text { and } \quad e^{\omega t}\left\|\kappa\left(P_{0} r_{0}, \eta\right)(t)\right\|_{F_{\mu}}
$$


uniformly for $t \geqslant 0$ using the property $\kappa\left(P_{0} r_{0}, \eta\right) \in \mathbb{F}_{1}\left(\mathbb{R}_{+}, \omega\right)$ and (5.10), respectively. Meanwhile, bounding integral terms in the $C(\mathbb{T})$-topology and using the bounds (5.9), we get

$$
\begin{gathered}
e^{\omega t} t^{1-\mu}\|r(t)-\eta\|_{E_{1}} \leqslant\left(1+c_{1} N\right)\left\|\kappa\left(P_{0} r_{0}, \eta\right)\right\|_{\mathbb{F}_{1}\left(\mathbb{R}_{+}, \omega\right)}, \quad t \geqslant 0, \\
e^{\omega t}\|r(t)-\eta\|_{E_{\mu}} \leqslant\left(c_{2}+c_{3} N\right)\left\|\kappa\left(P_{0} r_{0}, \eta\right)\right\|_{\mathbb{F}_{1}\left(\mathbb{R}_{+}, \omega\right)}, \quad t \geqslant 0 .
\end{gathered}
$$

Here the constant $c_{1}$ comes from the embedding $F_{1} \hookrightarrow F_{0}$, and the constants $c_{2}$ and $c_{3}$ come from the embeddings (5.10). Finally, by the regularity of $\kappa$, we may assume that $\tilde{U}$ was chosen sufficiently small to ensure that $D_{1} \kappa$ is uniformly bounded from $\tilde{U}$ into $\mathbb{F}_{1}\left(\mathbb{R}_{+}, \omega\right)$. Recalling that $\kappa(0, \eta)=0$, we have

$$
\begin{aligned}
\left\|\kappa\left(P_{0} r_{0}, \eta\right)\right\|_{\mathbb{F}_{1}\left(\mathbb{R}_{+}, \omega\right)} & \leqslant \int_{0}^{1}\left\|D_{1} \kappa\left(\tau P_{0} r_{0}, \eta\right) P_{0} r_{0}\right\|_{\mathbb{F}_{1}\left(\mathbb{R}_{+}, \omega\right)} d \tau \\
& \leqslant \tilde{M}\left\|P_{0} r_{0}\right\|_{F_{\mu}} \leqslant M\left\|r_{0}-r_{\star}\right\|_{E_{\mu}},
\end{aligned}
$$

where $M:=\left\|P_{0}\right\| \sup _{(\tilde{r}, \eta) \in \tilde{U}}\left\|D_{1} \kappa(\tilde{r}, \eta)\right\|_{\mathcal{L}\left(F_{\mu}, \mathbb{F}_{1}\left(\mathbb{R}_{+}, \omega\right)\right)}$. The claim now follows from (5.16) and the inequalities (5.14)-(5.15).

\subsection{Instability of cylinders with $0<r_{\star}<1$}

TheOREM 5.4 Let $r_{\star} \in(0,1)$ and $\mu \in[1 / 2,1]$ be fixed with $2 \mu+\alpha \notin \mathbb{Z}$. Then the equilibrium $r_{\star}$ of (2.4) is unstable in the topology of $h^{2 \mu+\alpha}(\mathbb{T})$ for initial values in $h^{2 \mu+\alpha}(\mathbb{T})$.

Proof. (i) Let $r_{\star} \in(0,1)$ be fixed, and let $L:=D G\left(r_{\star}\right)$. We can restate the evolution equation (2.4) in the following equivalent form

$$
\left\{\begin{array}{l}
\rho_{t}-L \rho=g(\rho), \quad t>0, \\
\rho(0)=r_{0}-r_{\star},
\end{array}\right.
$$

where $g(\rho):=G\left(\rho+r_{\star}\right)-L \rho$. Using the quasilinear structure of $G$ it is not difficult to see that for every $\beta>0$ there exists a number $\varepsilon_{0}=\varepsilon_{0}(\beta)>0$ so that

$$
\|g(\rho)\|_{E_{0}} \leqslant \beta\|\rho\|_{E_{1}}, \quad \text { for all } \quad \rho \in \mathbb{B}_{E_{\mu}}\left(0, \varepsilon_{0}\right) \cap E_{1} .
$$

It follows from (5.3) that

$$
\sigma(L) \cap[\operatorname{Re} z>0] \neq \emptyset,
$$

and we may choose numbers $\omega, \gamma>0$ such that

$$
[\omega-\gamma \leqslant \operatorname{Re} z \leqslant \omega+\gamma] \cap \sigma(L)=\emptyset \quad \text { and } \quad \sigma_{+}:=[\operatorname{Re} z>\omega+\gamma] \cap \sigma(L) \neq \emptyset .
$$

Define $P_{+}$to be the spectral projection, in $E_{0}$, with respect to the spectral set $\sigma_{+}$, and let $P_{-}:=$ $1-P_{+}$. Then $P_{+}\left(E_{0}\right)$ is finite dimensional and the topological decomposition

$$
E_{0}=P_{+}\left(E_{0}\right) \oplus P_{-}\left(E_{0}\right)
$$

reduces $L$. Hence, $L=L_{+} \oplus L_{-}$, where $L_{ \pm}$is the part of $L$ in $P_{ \pm}\left(E_{0}\right)$, respectively, with the domains $D\left(L_{ \pm}\right)=P_{ \pm}\left(E_{1}\right)$. Moreover, $P_{ \pm}$decomposes $E_{1}$ and, without loss of generality, we can take the norm on $E_{1}$ so that $\|v\|_{E_{1}}=\left\|P_{+} v\right\|_{E_{1}}+\left\|P_{-} v\right\|_{E_{1}}$. Note that

$$
\sigma\left(L_{-}\right) \subset[\operatorname{Re} z<\omega-\gamma], \quad \sigma\left(L_{+}\right)=\sigma^{+} \subset[\operatorname{Re} z>\omega+\gamma],
$$


which implies there is a constant $M_{0} \geqslant 1$ such that

$$
\begin{aligned}
\left\|e^{L_{-} t} P_{-}\right\|_{\mathcal{L}\left(E_{0}\right)} & \leqslant M_{0} e^{(\omega-\gamma) t}, \\
\left\|e^{-L_{+} t} P_{+}\right\|_{\mathcal{L}\left(E_{0}\right)} & \leqslant M_{0} e^{-(\omega+\gamma) t}, \quad t \geqslant 0
\end{aligned}
$$

where $\left\{e^{L_{-} t}: t \geqslant 0\right\}$ is the analytic semigroup in $P_{-}\left(E_{0}\right)$ generated by $L_{-}$and $\left\{e^{L_{+} t}: t \in \mathbb{R}\right\}$ is the group in $P_{+}\left(E_{0}\right)$ generated by the bounded operator $L_{+}$.

From [22, Theorem 5.2] one sees that $\left(\mathbb{E}_{0}(J), \mathbb{E}_{1}(J)\right)$ is a pair of maximal regularity for $-L$ and it is easy to see that $-L_{-}$inherits the property of maximal regularity. In particular, the pair $\left(P_{-}\left(\mathbb{E}_{0}(J)\right), P_{-}\left(\mathbb{E}_{1}(J)\right)\right)$ is a pair of maximal regularity for $-L_{-}$. In fact, since type $\left(-\omega+L_{-}\right)<$ $-\gamma<0$ we see that $\left(P_{-}\left(\mathbb{E}_{0}\left(\mathbb{R}_{+}\right)\right), P_{-}\left(\mathbb{E}_{1}\left(\mathbb{R}_{+}\right)\right)\right)$is a pair of maximal regularity for $\left(\omega-L_{-}\right)$. This, in turn, implies the a priori estimate

$$
\left\|e^{-\omega t} w\right\|_{\mathbb{E}_{1}\left(J_{T}\right)} \leqslant M_{1}\left(\left\|w_{0}\right\|_{E_{\mu}}+\left\|e^{-\omega t} f\right\|_{\mathbb{E}_{0}\left(J_{T}\right)}\right)
$$

for $J_{T}:=[0, T]$, any $T \in(0, \infty)$ (or $J_{T}=\mathbb{R}_{+}$for $T=\infty$ ), with a universal constant $M_{1}>0$, where $w$ is a solution of the linear Cauchy problem

$$
\left\{\begin{array}{l}
\dot{w}-L_{-} w=f, \\
w(0)=w_{0},
\end{array} \quad \text { with } \quad\left(f, w_{0}\right) \in\left(C\left((0, T), P_{-} E_{0}\right), P_{-} E_{0}\right) .\right.
$$

(ii) By way of contradiction, suppose that $r_{\star}$ is stable for (2.4). Then for every $\varepsilon>0$ there exists a number $\delta>0$ such that (5.17) admits for each $r_{0} \in \mathbb{B}_{E_{\mu}}\left(r_{\star}, \delta\right)$ a global solution $r=r\left(\cdot, r_{0}\right) \in$ $C_{1-\mu}^{1}\left(\mathbb{R}_{+}, E_{0}\right) \cap C_{1-\mu}\left(\mathbb{R}_{+}, E_{1}\right)$, which satisfies

$$
\|r(t)\|_{E_{\mu}}<\varepsilon, \quad t \geqslant 0 .
$$

We can assume without loss of generality that $\beta$ and $\varepsilon$ are chosen such that

$$
2 C_{0}\left(M_{0}+M_{1} \gamma\right) \beta \leqslant \gamma \quad \text { and } \varepsilon \leqslant \varepsilon_{0}(\beta),
$$

where $C_{0}:=\max \left\{\left\|P_{-}\right\|_{\mathcal{L}\left(E_{0}\right)},\left\|P_{+}\right\|_{\mathcal{L}\left(E_{0}\right)}\right\}$. As $P_{+}\left(E_{0}\right)$ is finite dimensional, we may also assume that

$$
\left\|P_{+} v\right\|_{E_{v}}=\left\|P_{+} v\right\|_{E_{0}}, \quad v \in E_{0}, \quad v \in\{\mu, 1\},
$$

using the fact that $P_{+} E_{0} \subset D\left(L^{n}\right)$ for every $n \in \mathbb{N}$, cf. [24, Proposition A.1.2].

Claim 1: For any initial value $r_{0} \in \mathbb{B}_{E_{\mu}}\left(r_{\star}, \delta\right), P_{+} r$ admits the representation

$$
P_{+} r(t)=-\int_{t}^{\infty} e^{L_{+}(t-s)} P_{+} g(r(s)) d s, \quad t \geqslant 0 .
$$

For this we first establish that, for $r_{0} \in \mathbb{B}_{E_{\mu}}\left(r_{\star}, \delta\right)$,

$$
e^{-\omega t} r \in B C_{1-\mu}\left(\mathbb{R}_{+}, E_{1}\right):=\left\{u \in C\left((0, \infty), E_{1}\right): \sup _{t \in \mathbb{R}_{+}} t^{1-\mu}\|u(t)\|_{E_{1}}<\infty\right\} .
$$


The mapping property $g: \mathbb{E}_{1}\left(J_{T}\right) \rightarrow \mathbb{E}_{0}\left(J_{T}\right)$ follows analogously to the mapping property derived for $\mathcal{G}$ in the proof of Theorem 5.3 above, $0<T<\infty$. Together with the inequalities (5.18) and (5.20), this yields

$$
\begin{aligned}
\left\|e^{-\omega t} P_{-} r\right\|_{B_{1-\mu}\left(J_{T}, E_{1}\right)} & \leqslant M_{1}\left(\left\|P_{-}\left(r_{0}-r_{\star}\right)\right\|_{E_{\mu}}\right. \\
& \left.+C_{0} \beta\left\|e^{-\omega t} P_{+} r\right\|_{B_{1-\mu}\left(J_{T}, E_{1}\right)}+C_{0} \beta\left\|e^{-\omega t} P_{-} r\right\|_{B_{1-\mu}\left(J_{T}, E_{1}\right)}\right)
\end{aligned}
$$

for any $0<T<\infty$. Due to (5.22), we have $M_{1} C_{0} \beta \leqslant 1 / 2$ and can further conclude

$$
\left\|e^{-\omega t} P_{-} r\right\|_{B_{1-\mu}\left(J_{T}, E_{1}\right)} \leqslant 2 M_{1}\left(\left\|P_{-}\left(r_{0}-r_{\star}\right)\right\|_{E_{\mu}}+C_{0} \beta\left\|e^{-\omega t} P_{+} r\right\|_{B_{1-\mu}\left(J_{T}, E_{1}\right)}\right) .
$$

It follows from (5.21) that

$$
t^{1-\mu}\left\|e^{-\omega t} P_{+} r(t)\right\|_{E_{1}} \leqslant t^{1-\mu} e^{-\omega t} C_{0}\|r(t)\|_{E_{\mu}} \leqslant C_{0} C_{1} \varepsilon
$$

where $C_{1}:=\sup \left\{t^{1-\mu} e^{-\omega t}: t \geqslant 0\right\}<\infty$. Inserting this result into (5.25) yields

$$
\left\|e^{-\omega t} r\right\|_{B_{1-\mu}\left(J_{T}, E_{1}\right)} \leqslant 2 M_{1}\left\|P_{-}\left(r_{0}-r_{\star}\right)\right\|_{E_{\mu}}+\left(2 M_{1} C_{0} \beta+1\right) C_{0} C_{1} \varepsilon \leqslant C_{2}
$$

for any $0<T<\infty$. However, since $T$ is arbitrary and (5.26) is independent of $T$ we conclude that $e^{-\omega t} r \in B C_{1-\mu}\left(\mathbb{R}_{+}, E_{1}\right)$, for any initial value $r_{0} \in \mathbb{B}_{E_{\mu}}\left(r_{\star}, \delta\right)$. Next we note that, for $s \geqslant t$, by (5.19)

$$
\begin{aligned}
\left\|e^{L_{+}(t-s)} P_{+} g(r(s))\right\|_{E_{0}} & \leqslant M_{0} C_{0} \beta e^{(\omega+\gamma)(t-s)}\|r(s)\|_{E_{1}} \\
& \leqslant M_{0} C_{0} \beta e^{\omega t} e^{\gamma(t-s)} s^{\mu-1}\left\|e^{-\omega s} r\right\|_{B_{1-\mu}\left(\mathbb{R}_{+}, E_{1}\right)}
\end{aligned}
$$

by which the integral in (5.23) exists for $t \geqslant 0$, convergence in $E_{1}$. Moreover,

$$
\left\|\int_{t}^{\infty} e^{L_{+}(t-s)} P_{+} g(r(s)) d s\right\|_{E_{0}} \leqslant e^{\omega t} M_{0} C_{0} C_{3} \beta\left\|e^{-\omega t} r\right\|_{B_{1-\mu}\left(\mathbb{R}_{+}, E_{1}\right)},
$$

where $C_{3}:=\sup \left\{\int_{t}^{\infty} e^{\gamma(t-s)} s^{\mu-1} d s: t \geqslant 0\right\}<\infty$. Noting that $w=P_{+} r$ solves the Cauchy problem

$$
\left\{\begin{array}{l}
\dot{w}-L_{+} w=P_{+} g(r), \\
w(0)=P_{+}\left(r_{0}-r_{\star}\right),
\end{array}\right.
$$

it follows from the variation of parameters formula that, for $t \geqslant 0$ and $\tau>0$,

$$
P_{+} r(t)=e^{L_{+}(t-\tau)} P_{+} r(\tau)+\int_{\tau}^{t} e^{L_{+}(t-s)} P_{+} g(r(s)) d s .
$$

This representation holds for any $\tau>0$ and the claim follows from (5.19) and (5.21) by sending $\tau$ to $\infty$.

CLAim 2: If $r_{0} \in \mathbb{B}_{E_{\mu}}\left(r_{\star}, \delta\right)$ and $\|r(t)\|_{E_{\mu}}<\varepsilon$ for all $t \geqslant 0$, then

$$
\left\|P_{+}\left(r_{0}-r_{\star}\right)\right\|_{E_{\mu}} \leqslant 2 M_{0} M_{1} C_{3}\left\|P_{-}\left(r_{0}-r_{\star}\right)\right\|_{E_{\mu}} .
$$


From (5.23) and (5.27) follows

$$
\left\|e^{-\omega t} P_{+} r\right\|_{B_{1-\mu}\left(\mathbb{R}_{+}, E_{0}\right)} \leqslant \frac{M_{0} C_{0} \beta}{\gamma}\left(\left\|e^{-\omega t} P_{+} r\right\|_{B_{1-\mu}\left(\mathbb{R}_{+}, E_{1}\right)}+\left\|e^{-\omega t} P_{-} r\right\|_{B_{1-\mu}\left(\mathbb{R}_{+}, E_{1}\right)}\right)
$$

where we have used the fact that $\sup _{t \geqslant 0}\left\{t^{1-\mu} \int_{t}^{\infty} e^{\gamma(t-s)} s^{\mu-1} d s\right\} \leqslant 1 / \gamma$. Adding the estimates in (5.24) and (5.29) and employing (5.22) yields

$$
\left\|e^{-\omega t} r\right\|_{B_{1-\mu}\left(\mathbb{R}_{+}, E_{1}\right)} \leqslant 2 M_{1}\left\|P_{-}\left(r_{0}-r_{\star}\right)\right\|_{E_{\mu}} .
$$

The representation (5.23) in conjunction with (5.28) and (5.30) then implies

$$
\left\|P_{+}\left(r_{0}-r_{\star}\right)\right\|_{E_{\mu}} \leqslant M_{0} C_{0} C_{3} \beta\left\|e^{-\omega t} r\right\|_{B_{1-\mu}\left(\mathbb{R}_{+}, E_{1}\right)} \leqslant M_{0} C_{3}\left\|P_{-}\left(r_{0}-r_{\star}\right)\right\|_{E_{\mu}},
$$

where the last inequality follows from $2 C_{0} M_{1} \beta \leqslant 1$. We have thus demonstrated the claim, and the theorem follows by way of contradiction. In particular, note that if $r_{0} \in \mathbb{B}_{E_{\mu}}\left(r_{\star}, \delta\right)$ is chosen with $\left\|P_{-}\left(r_{0}-r_{\star}\right)\right\|_{E_{\mu}}=0$, then it must hold that $\left\|P_{+}\left(r_{0}-r_{\star}\right)\right\|_{E_{\mu}}=0$, so that $r_{0}=r_{\star}$, which contradicts the stability assumption.

\section{Bifurcation from cylinders}

We conclude by investigating the interactions between the family of cylinders, which we will informally consider as the trivial equilibria of (2.4), and the families of undulary curves, the nontrivial equilibria. In particular, by restricting (5.7) to a problem on profile functions which are symmetric about the $y$-axis (i.e. even functions on $\mathbb{T}$ ), we observe subcritical bifurcations from the family of cylinders which occur at the cylinder of radius $1 / \ell$, for any $\ell \in \mathbb{N}$, where the reciprocal of the radius $r_{\star}$ is taken as a bifurcation parameter. Most of the terminology and notations employed in this section coincide with those of Kielhöfer [21].

To begin, define the operator

$$
\bar{G}(\tilde{r}, \lambda):=P_{0} G\left(\psi_{\star}\left(\tilde{r}, r_{\star}\right)\right), \quad \lambda:=1 / r_{\star}, r_{\star}>0,
$$

and we immediately note that

$$
\bar{g} \in C^{\omega}\left(\left(h_{0, e}^{2+\alpha}(\mathbb{T}) \cap U_{0}\right) \times(0, \infty), h_{0, e}^{\alpha}(\mathbb{T})\right),
$$

where $F_{j, e}:=h_{0, e}^{2 j+\alpha}(\mathbb{T})$ denotes the space of even, zero-mean functions with $h^{2 j+\alpha}$-regularity over $\mathbb{T}, j=0,1$. We observe that $\bar{g}(\cdot, \lambda)$ preserves symmetry about the $y$-axis, a property that follows quickly from the definition (2.3) of the governing operator $G$ and the representation of $\psi_{\star}$ derived in Remarks 5.1(f). The fact that $G$ preserves even functions is easily noted by the fact that every term of $G(r)$ is either constant on $\mathbb{T}$ due to integration, or depends only on $r, r_{x x}$, and $r_{x}^{2}$, which are each even functions on $\mathbb{T}$ when $r$ is itself taken to be even on $\mathbb{T}$.

TheOREM 6.1 Fix $\ell \in \mathbb{N}$. Then $(0, \ell)$ is a bifurcation point for the equation

$$
\overline{\mathrm{G}}(\tilde{r}, \lambda)=0, \quad(\tilde{r}, \lambda) \in h_{0, e}^{2+\alpha}(\mathbb{T}) \times(0, \infty) .
$$

In particular, there exists a positive constant $\delta_{\ell}>0$ and a nontrivial analytic curve

$$
\left\{\left(\tilde{r}_{\ell}(s), \lambda_{\ell}(s)\right) \in h_{0, e}^{2+\alpha}(\mathbb{T}) \times \mathbb{R}: s \in\left(-\delta_{\ell}, \delta_{\ell}\right),\left(\tilde{r}_{\ell}(0), \lambda_{\ell}(0)\right)=(0, \ell)\right\},
$$


such that

$$
\overline{\mathrm{g}}\left(\tilde{r}_{\ell}(s), \lambda_{\ell}(s)\right)=0 \text { for all } s \in\left(-\delta_{\ell}, \delta_{\ell}\right),
$$

and all solutions of $(6.1)$ in a neighborhood of $(0, \ell)$ are either a trivial solution $(0, \lambda)$ or an element of the nontrivial curve (6.2). Moreover, if $\lambda \in \mathbb{R}_{+} \backslash \mathbb{N}$, then $(0, \lambda)$ is not a bifurcation point for (6.1). We can further conclude that

a. lifting the curve (6.2), via $\psi_{\star}$, we get elements of the family of $2 \pi / \ell$-periodic undulary curves with parameter values $|B|<\tilde{\delta}_{\ell}$ for some $\tilde{\delta}_{\ell}>0$.

b. the bifurcation is a subcritical pitchfork type bifurcation. More precisely, for all $\ell \in \mathbb{N}$, we have

$$
\dot{\lambda}_{\ell}(0)=0 \quad \text { and } \quad \ddot{\lambda}_{\ell}(0)<0,
$$

where " " " denotes differentiation with respect to the parameter $s$.

c. the bifurcating branch of undulary curves are unstable equilibria of (2.4), at least for parameter values $|B|<\tilde{\delta}_{\ell}$ sufficiently small.

Proof. By working in the setting of even functions on $\mathbb{T}$, we can take advantage of the cosineFourier series representation

$$
\tilde{r}(x)=\sum_{k \geqslant 1} \hat{\tilde{r}}(k) \cos (k x), \quad \text { for all } \quad \tilde{r} \in h_{0, e}^{\sigma}(\mathbb{T}),
$$

where $\hat{\tilde{r}}(k)=\frac{1}{\pi} \int_{\mathbb{T}} \tilde{r}(x) \cos (k x) d x$ are the cosine-Fourier coefficients of $\tilde{r} \in h_{0, e}^{\sigma}(\mathbb{T}), k \geqslant 1$. It follows easily from (5.12) that the linearization $D_{1} \overline{\mathrm{g}}(0, \lambda)$ is a Fourier multiplier with symbol

$$
\left(M_{k}\right)_{k \geqslant 1}:=\left(\lambda^{2}-k^{2}\right)_{k \geqslant 1} .
$$

We then see that $D_{1} \bar{g}(0, \lambda)$ is bijective from $F_{1, e}$ onto $F_{0, e}$ when $\lambda \in \mathbb{R}_{+} \backslash \mathbb{N}$, so that only points of the form $(0, \ell)$ can possibly be bifurcation points for $(6.1)$.

Proceeding to verify that $(0, \ell)$ is indeed a bifurcation point, we compute the kernel and range of $D_{1} \overline{\mathrm{g}}(0, \ell)$ as

$$
N_{\ell}=\operatorname{span}\{\cos (\ell x)\} \quad \text { and } \quad R_{\ell}=\overline{\operatorname{span}}\{\cos (k x): k \geqslant 1, k \neq \ell\},
$$

respectively. Since $h^{\sigma}(\mathbb{T}) \hookrightarrow L_{2}(\mathbb{T})$, we can borrow the $L_{2}$-inner product to realize $N_{\ell}$ as a topological complement to $R_{\ell}$ as subspaces of $F_{1, e}$. By compactness of the resolvent $R(\lambda):=$ $\left(\lambda-D G\left(r_{\star}\right)\right)^{-1}, \lambda \in \rho\left(D G\left(r_{\star}\right)\right)$, it follows that $D_{1} \bar{g}(0, \ell)$ is a Fredholm operator of index zero. Then, defining the element $\hat{v}_{0}:=\cos (\ell x)$, and noting that the second-order mixed partial (Fréchet) derivative ${ }^{1} D_{12}^{2} \bar{g}(\tilde{r}, \lambda)$ satisfies

$$
D_{12}^{2} \bar{g}(0, \ell) \hat{v}_{0}=-2 \ell P_{0} \hat{v}_{0}=-2 \ell \cos (\ell x) \notin R_{\ell},
$$

it follows by [8, Theorem 1.7], or [21, Theorem I.5.1], that (6.1) bifurcates at $(0, \ell)$, which proves the main part of the theorem.

\footnotetext{
${ }^{1}$ By definition, the second order (mixed) derivative $D_{12}^{2} \bar{g}$ maps $\left(h_{0, e}^{2+\alpha}(\mathbb{T}) \cap U_{0}\right) \times(0, \infty)$ into the bilinear operators $\mathcal{L}\left(h_{0, e}^{2+\alpha}(\mathbb{T}) \times \mathbb{R}, h_{0, e}^{\alpha}(\mathbb{T})\right)$. However, it is customary to contract along partial derivatives with respect $\mathbb{R}$ valued variables. Hence, we identify $D_{12}^{2} \overline{\mathcal{G}}(\tilde{r}, \lambda)(\cdot, \cdot)$ with the linear operator $D_{12}^{2} \overline{\mathcal{G}}(\tilde{r}, \lambda) \cdot=D_{12}^{2} \overline{\mathcal{G}}(\tilde{r}, \lambda)(\cdot, 1) \in$ $\mathcal{L}\left(h_{0, e}^{2+\alpha}(\mathbb{T}), h_{0, e}^{\alpha}(\mathbb{T})\right)$, cf. [8].
} 
(a) Notice that $\psi_{\star}(0, \ell)$ is precisely the constant function $r_{\star}=1 / \ell$, and, utilizing the explicit characterization of equilibria from Section 4 above, we know that a curve of non-trivial equilibria for (2.4) containing this function must coincide with the family of $\frac{2 \pi}{\ell}$-periodic unduloids. Hence, it suffices to show that $\bar{g}(\tilde{r}, \lambda)=0$ if and only if $G\left(\psi_{\star}\left(\tilde{r}, \lambda^{-1}\right)\right)=0$, i.e. $\psi_{\star}\left(\tilde{r}, \lambda^{-1}\right)$ is an equilibrium of (2.4) if and only if $(\tilde{r}, \lambda)$ solves (6.1). However, this follows immediately from the relation

$$
G(r)=D_{1} \psi_{\star}\left(P_{0} r, \eta\right) P_{0} G(r), \quad r \in E_{1}
$$

which was justified in deriving (5.11) above.

(b) We will follow the characterization of bifurcation types as developed in [21, Sections I.6 and I.7]. Evaluating the second-order derivative

$$
\begin{aligned}
D_{11}^{2} \overline{\mathrm{g}}(0, \ell)\left[\hat{v}_{0}, \hat{v}_{0}\right]= & P_{0}\left(2 \ell^{4} Q_{0}\left(\cos ^{2}(\ell x)\right)-2 \ell^{3} P_{0}\left(\cos ^{2}(\ell x)\right)-\ell^{4} Q_{0}\left(\sin ^{2}(\ell x)\right)\right. \\
& \left.-\ell^{3} \sin ^{2}(\ell x)+2 \ell^{3} Q_{0}\left(\cos ^{2}(\ell x)\right)\right) \\
= & -\ell^{3} P_{0}\left(\frac{\cos (2 \ell x)+1}{2}\right)=-\ell^{3} / 2 \cos (2 \ell x),
\end{aligned}
$$

we note that $-\ell^{3} / 2 \cos (2 \ell x) \in R_{\ell}$, from which it follows that $\dot{\lambda}(0)=0$. Meanwhile, utilizing the representation [21, (I.6.11) and (I.6.9)] and following a considerable amount of computation, one will see that

$$
\ddot{\lambda}(0)=-\frac{1}{3} \frac{\left\langle 6 \ell^{4} \hat{v}_{0}, \hat{v}_{0}\right\rangle}{\left\langle 2 \ell \hat{v}_{0}, \hat{v}_{0}\right\rangle}=-\ell^{3}<0,
$$

where $\langle\cdot, \cdot\rangle$ denotes the inner product on $L^{2}(\mathbb{T})$, within which $h_{0, e}^{\sigma}(\mathbb{T})$ is embedded.

(c) We can now track the so-called critical eigenvalue $\mu_{\ell}(\lambda)$ of the linearization $D_{1} \bar{g}(0, \lambda)$, which is the eigenvalue which passes through 0 at $\lambda=\ell$, with non-vanishing speed, as guaranteed by the observed bifurcation. Moreover, via eigenvalue perturbation techniques, we can also track the perturbed eigenvalues $\hat{\mu}_{\ell}(s)$ of the linearization $D_{1} \overline{\mathcal{G}}\left(\tilde{r}_{\ell}(s), \lambda_{\ell}(s)\right)$. Then, by taking a derivative of the relation (6.3), we observe that

$$
D G\left(\psi_{\star}(\tilde{r}, \eta)\right) D_{1} \psi_{\star}(\tilde{r}, \eta) \tilde{h}=D_{1} \psi_{\star}(\tilde{r}, \eta) D_{1} G(\tilde{r}, \eta) \tilde{h}, \quad \tilde{h} \in F_{1},
$$

from which one will easily conclude that $\hat{\mu}(s)$ must also be an eigenvalue of the linearization $D G\left(\psi_{\star}\left(\tilde{r}_{\ell}(s), \lambda_{\ell}^{-1}(s)\right)\right.$. Finally, by the subcritical structure of the bifurcation, we conclude that, for sufficiently small $|s|<\delta_{\ell}$, the perturbed eigenvalue $\hat{\mu}_{\ell}(s)$ has positive real part, from which instability follows by a similar argument to Theorem 5.4 above.

REMARK 6.2 If one wishes to generalize the axisymmetric averaged mean curvature flow to the setting of arbitrary periodicity, all results contained herein continue to hold, with minor modifications. For the reader's convenience, we mention here some details to reformulate our results in the $p$-periodic setting.

Letting $p>0$, we denote by $\mathbb{T}_{p}:=[-p / 2, p / 2]$ the interval of $p$-periodicity and $h^{k+\alpha}\left(\mathbb{T}_{p}\right)$ the regular functions on $\mathbb{T}_{p}$, analogous to $h^{k+\alpha}(\mathbb{T})$. To formulate (2.4), one makes obvious modifications to the non-local quantities $S(r)$ and $h(r)$, where integration is now taken over the interval $\mathbb{T}_{p}$. Meanwhile, the local quantities $V(t)$ and $\mathcal{H}(r)$ remain unchanged in structure, as 
applied to a profile function $r: \mathbb{T}_{p} \rightarrow(0, \infty)$. The structure and well-posedness of the problem remain essentially unchanged, so we turn our attention to the equilibria and bifurcation behavior.

It is clear that the only equilibria for the $p$-periodic version of (2.4) remain the cylinders and the $p$-periodic unduloids. The primary difference one observes in this setting is the fact that the unduloids (first) bifurcate from the family of cylinders at the critical radius $r_{\star}=p / 2 \pi$ and subsequent bifurcations occur at radii

$$
r_{\ell}=\frac{p}{2 \pi \ell}, \quad \ell \in \mathbb{N}
$$

This observation follows from a natural extension of the methods of the paper to the $p$-periodic domain and the following:

a. To enforce $p$-periodicity of the unduloids, as expressed in Theorem 4.1, one must have the relationship

$$
\frac{p H}{k}=\int_{\pi / 2}^{5 \pi / 2} \frac{1+B \sin t}{\sqrt{1+B^{2}+2 B \sin t}} d t
$$

which is the analogue of (4.1). Noting that $B=0$ corresponds to a cylinder in the family of parametrized unduloids, this relation tells us the associated cylinders are exactly those with mean curvature $\mathcal{H}=2 \pi k / p$. Which aligns precisely with the cylinders of radius $r_{k}=p / 2 \pi k$, recalling that mean curvature of a cylinder is always reciprocal of the radius.

b. To characterize the spectrum of the linearization $D G\left(r_{\star}\right)$, given analogous to (5.1), one utilizes the Fourier series characterization of functions $r \in h^{\sigma}\left(\mathbb{T}_{p}\right)$, which reads

$$
r(x)=\sum_{k \in \mathbb{Z}} \hat{r}(k) e^{i k x 2 \pi / p}, \quad x \in \mathbb{T}_{p} ; \quad \text { where } \hat{r}(k):=\frac{1}{p} \int_{\mathbb{T}_{p}} r(x) e^{-i k x 2 \pi / p} d x .
$$

Thus, one arrives at spectrum

$$
\sigma\left(D G\left(r_{\star}\right)\right)=\{0\} \cup\left\{\left(r_{\star}^{-2}-(2 \pi k / p)^{2}\right): k \in \mathbb{Z} \backslash\{0\}\right\},
$$

from which all results regarding stability, instability, and bifurcation follow in the same manner as the $2 \pi$-periodic setting.

Acknowledgments. The author thanks Professor Gieri Simonett for introducing him to geometric evolution equations and for providing many helpful conversations and suggestions in the preparation of this manuscript. The author also thanks the anonymous referees for helpful suggestions that have improved the applicability of the presentation.

\section{REFERENCES}

1. Amann, H., Linear and Quasilinear Parabolic Problems, Monographs in Mathematics vol. 89. Birkhäuser Verlag, Basel (1995). Zb10819.35001 MR1345385

2. Amann, H., Quasilinear parabolic problems via maximal regularity. Adv. Differential Equations 10 (2005), 1081-1110. Zbl 103.35059 MR2162362

3. Angenent, S., Nonlinear analytic semiflows. Proc. of the Royal Soc. of Edinburgh 115A (1990), 91107. Zb10723.34047 MR1059647

4. Angenent, S., Parabolic equations for curves on surfaces. I. Curves with p-integrable curvature. Ann. of Math. (2) 132 (1990), 451-483. Zb10789. 58070 MR1078266 
5. Athanassenas, M., Volume-preserving mean curvature flow of rotationally symmetric surfaces. Comment. Math. Helv. 72 (1997), 52-66. Zbl0873. 35033 MR1456315

6. CABEZAS-Rivas, E., \& MiQuel, V., Volume-preserving mean curvature flow of revolution hypersurfaces in a rotationally symmetric space. Math. Z. 261 (2009), 489-510. Zbl1161.53053 MR2471083

7. Clément, P., \& Simonett, G., Maximal regularity in continuous interpolation spaces and quasilinear parabolic equations. J. Evol. Equ. 1 (2001), 39-67. Zb10988. 35099 MR1838320

8. Crandall, M., \& Rabinowitz, P., Bifurcation from simple eigenvalues. J. Functional Analysis 8 (1971), 321-340. Zb10219.46015 MR0288640

9. Da Prato, G., \& Grisvard, P., Equations d'évolution abstraites non linéaires de type parabolique. Ann. Mat. Pura Appl. (4) 120 (1979), 329-396. Zbl0471. 35036 MR0551075

10. Deimling, K., Nonlinear Functional Analysis. Springer-Verlag, Berlin (1985). Zb10559.47040 MR0787404

11. Delaunay, C., Sur la surface de révolution dont la courbure moyenne est constante. J. Math. Pures. Appl. Ser. 16 (1841), 309-320.

12. Escher, J., Prüss, J., \& Simonett, G., A new approach to the regularity of solutions for parabolic equations. In: Evolution Equations. Lecture Notes in Pure and Appl. Math. 234, Dekker, New York (2003), 167-190. Zbl1070.35009 MR2073744

13. Escher, J., \& Simonett, G., Analyticity of the interface in a free boundary problem. Math. Ann. 305 (1996), 439-459. Zb10857.76086 MR1397432

14. Escher, J., \& Simonett, G., The volume preserving mean curvature flow near spheres. Proc. Amer. Math. Soc. 126 (1998), 2789-2796. Zb10909.53043 MR1485470

15. Gage, M., On an area-preserving evolution equation for plane curves. In: Nonlinear Problems in Geometry (Mobile, AL, 1985), Contemp. Math. 51, Amer. Math. Soc., Providence (1986), 51-62. Zb10608.53002 MR0848933

16. Gage, M., \& Hamilton, R., The heat equation shrinking convex plane curves. J. Diff. Geom. 23 (1986), 69-96. Zb10621.53001 MR0840401

17. Hartley, D., Motion by volume preserving mean curvature flow near cylinders. arXiv:1205.0339.

18. Huisken, G., The volume preserving mean curvature flow. J. Reine Angew. Math. 382 (1987), 35-48. Zbl0621.53007 MR0921165

19. Huisken, G., \& Polden, A., Geometric evolution equations for hypersurfaces. In: Calculus of variations and geometric evolution problems (Cetraro, 1996), Lecture Notes in Math. 1713, Springer, Berlin (1999), 45-84. Zb10942.35047 MR1731639

20. Kenmotsu, K., Surfaces of revolution with prescribed mean curvature. Tôhoku Math. J. (2) 32 (1980), 147-153. Zbl0431.53005 MR0567837

21. KIELHÖFER, H., Bifurcation Theory. An Introduction With Applications to Partial Differential Equations. 2nd ed., Applied Mathematical Sciences, 156. Springer, New York (2012). Zbl1230. 35002 MR2859263

22. LeCrone, J., Elliptic operators and maximal regularity on periodic little-Hölder spaces. J. Evol. Equ. 12 (2012), 295-325. Zbl1258.35132 MR2923936

23. LeCrone, J., \& Simonett, G., On well-posedness, stability and bifurcation for the axisymmetric surface diffusion flow. SIAM J. Math. Anal. 45 (2013), 2834-2869. Zbl 06248245 MR3103249

24. Lunardi, A., Analytic Semigroups and Optimal Regularity in Parabolic Problems. Birkhäuser, Basel (1995). Zb10816.35001 MR1329547

25. MASUDA, K., On the regularity of solutions of the nonstationary Navier-Stokes equations. In: Approximation methods for Navier-Stokes problems. (Paderborn, 1979). Lecture Notes in Math. 771, Springer, Berlin (1980), 360-370. Zbl0435.35029 MR0566007

26. Mayer, U., \& Simonett, G., Self-intersection for the surface diffusion and the volume-preserving mean curvature flow. Differential Integral Equations 13 (2000), 1189-1199. Zb11013. 53045 MR1775252 
27. Prokert, G., Parabolic evolution equations for quasistationary free boundary problems in capillary fluid mechanics. PhD Thesis, Technische Univ. Eindhoven (1997). Zb10896 . 35145 MR1454616

28. PrÜSS, J., Maximal regularity for evolution equations in $L_{p}$-spaces. Conf. Semin. Mat. Univ. Bari 285 (2003), 1-39.

29. Prüss, J., Simonett, G., \& Zacher, R., On convergence of solutions to equilibria for quasilinear parabolic problems. J. Differential Equations 246 (2009), 3902-3931. Zbl1172 . 35010 MR2514730

30. Vondenhoff, E., Long-time asymptotics of Hele-Shaw flow for perturbed balls with injection and suction. Interfaces Free Bound. 10 (2008), 483-502. Zbl1154. 35481 MR2465270 